\title{
Finite-volume scheme for anisotropic diffusion
}

\author{
Bram van Es ${ }^{a, b, *}$, Barry Koren ${ }^{c}$, Hugo J. de Blank ${ }^{b}$ \\ a Centrum Wiskunde E Informatica, P.O. Box 94079, 1090GB Amsterdam, The Netherlands \\ ${ }^{\mathrm{b}}$ FOM Institute DIFFER, Dutch Institute for Fundamental Energy Research, The Netherlands ${ }^{1}$ \\ c Eindhoven University of Technology, The Netherlands
}

\section{A R T I C L E I N F O}

Article history:

Received 29 September 2014

Received in revised form 3 October 2015

Accepted 20 November 2015

Available online 2 December 2015

\section{Keywords:}

Finite volumes

Anisotropic diffusion

\begin{abstract}
A B S T R A C T
In this paper, we apply a special finite-volume scheme, limited to smooth temperature distributions and Cartesian grids, to test the importance of connectivity of the finite volumes. The area of application is nuclear fusion plasma with field line aligned temperature gradients and extreme anisotropy. We apply the scheme to the anisotropic heat-conduction equation, and compare its results with those of existing finite-volume schemes for anisotropic diffusion. Also, we introduce a general model adaptation of the steady diffusion equation for extremely anisotropic diffusion problems with closed field lines.
\end{abstract}

(c) 2015 Elsevier Inc. All rights reserved.

\section{Introduction}

Most of the techniques to handle diffusion in anisotropic media are based on finite-volume or finite-element methods and revolve around handling the interpolation of the flux over the cell faces. A lot of work has been done on finite-volume schemes for the solution of diffusion problems on unstructured grids with discontinuous and anisotropic diffusion tensors. Here an important assumption in constructing the formulation of the cell-face fluxes is the continuity of the heat flux over the cell-faces, see for instance Edwards and Rogers [13], Breil and Maire [31] and Jacq et al. [25]. Vertex values are used in several cell-centered schemes to approximate the flux over the cell face, see e.g. Le Potier [28], Lipnikov et al. [30], Coudière et al. [10]. The vertex values are approximated with for instance continuity and monotonicity in mind. The vertex values may be defined explicitly but this requires some sort of dual grid, see e.g. Hermeline [22], Le Potier and Ong [29], Morel et al. [32]. Shashkov and Steinberg [34] put the flux values in the vertices and then average to the centers of the cell-faces. For a more detailed overview of finite-volume methods the reader is referred to the review paper by Droniou [11].

One of the most popular finite-volume methods for diffusion problems is the Multi-Point Flux Approximation (MPFA), a cell-centered finite-volume method commonly used for approximating diffusion with discontinuous tensors on distorted meshes, see e.g. Aavatsmark et al. [1,3-6] and Edwards and Rogers [13]. The method is robust in terms of diffusion tensor discontinuity. However, the resulting diffusion operator is often non-symmetric and formal accuracy cannot be maintained for higher levels of anisotropy. Aavatsmark [2] present a symmetric MPFA method and give formal proof of convergence. Friis et al. [20] and Edwards and Pal [12] present symmetric MPFA schemes for unstructured triangular and quadrilateral volumes respectively. The MPFA-method is also applied to multi-phase, multi-scale diffusion problems with grid refinement by Jenny et al. [26,27] and Hesse et al. [23].

\footnotetext{
* Corresponding author.

E-mail address: bramiozo@gmail.com (B. van Es).

1 www.differ.nl.
} 


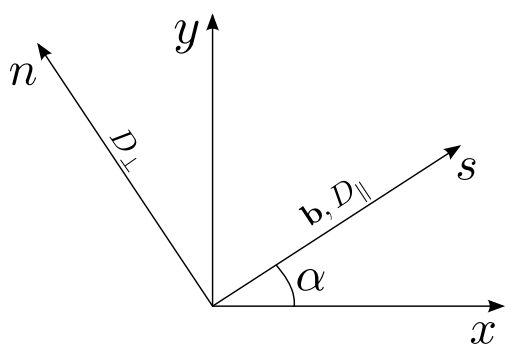

Fig. 1. Explanation of symbols.

Edwards and Zheng [14,16], Chen et al. [9] and Friis and Edwards [19] use a flux-continuous locally conservative MPFAscheme called CVD-MPFA. The CVD-MPFA scheme includes the full diffusion tensor and extra auxiliary vertex unknowns and interface pressure value that are eliminated a priori using an auxiliary divergence condition and flux continuity conditions. They use M-matrix conditions and temperature continuity and flux continuity conditions to obtain a suitable quadrature point for the flux formulation. In case an M-matrix cannot be constructed, a Quasi-M-matrix is constructed which fulfills the former conditions as much as possible. The method does not guarantee M-matrices for high values of anisotropy, the latter is improved in the double parameter family of this scheme by Edwards and Zheng [15] and more generally the multi-parameter family by Edwards and Zheng [17]. These methods are robust for general tensor fields and unstructured grids.

What motivated Morel et al. [32], Breil and Maire [7], Hyman et al. [24], Edwards and Zheng [14,16] and others in developing flux(-normal) continuous schemes was grid robustness of finite-volume methods and finite-element methods in case of diffusion-tensor discontinuities. Van Es et al. [37] looked at the importance of alignment for a finite-difference method. In that paper several schemes are compared. The importance of internodal/volume continuity was expected because the formal accuracy for all schemes using series expansions was second order and a decisive effect of lower continuity at the boundaries was not visible in a local error analysis although it clearly mattered in terms of boundary treatment.

In this paper we propose and apply a finite-volume scheme that can change the connectivity between the volumes by changing the length of the cell faces with a free parameter. We apply both cell-face and vertex-centered flux points.

As before we approximate the anisotropic thermal diffusion, described by

$$
\mathbf{q}=-\mathbf{D} \cdot \nabla T, \quad \frac{\partial T}{\partial t}=-\nabla \cdot \mathbf{q}+f,
$$

where $T$ represents the temperature, $\mathbf{b}$ the unit direction vector of the field line, $f$ some source term and $\mathbf{D}$ the diffusion tensor. For a two-dimensional problem the diffusion tensor is given by

$$
\begin{array}{ll}
\text { unit direction vector: } & \mathbf{b}=(\cos \alpha, \sin \alpha)^{T}, \\
& \mathbf{D}=\left(D_{\|}-D_{\perp}\right) \mathbf{b b}^{T}+D_{\perp} \mathcal{I}, \\
& \mathbf{D}=\left(\begin{array}{cc}
D_{\|} b_{1}^{2}+D_{\perp} b_{2}^{2} & \left(D_{\|}-D_{\perp}\right) b_{1} b_{2} \\
\left(D_{\|}-D_{\perp}\right) b_{1} b_{2} & D_{\perp} b_{1}^{2}+D_{\|} b_{2}^{2}
\end{array}\right),
\end{array}
$$

where $D_{\|}$and $D_{\perp}$ represent the parallel and the perpendicular diffusion coefficient respectively.

We define $x, y$ as the non-aligned coordinate system and $s, n$ as the aligned coordinate system, see Fig. 1 . We define the anisotropy as

$$
\varsigma=\frac{D_{\|}}{D_{\perp}} .
$$

In nuclear fusion plasma the level of anisotropy $\zeta$ can be as large as $10^{9}$, i.e. the diffusivity along the field lines is in the order of $10^{9}$ times larger than the diffusivity perpendicular to the field lines. As the directions of the magnetic field lines vary continuously we have a full diffusion tensor throughout the domain. Variability of the diffusion coefficients is not considered in the current work.

\section{Finite-volume schemes}

All the schemes to be discussed formally have local second-order accuracy, which can be shown by carefully expanding the approximations using Taylor series, see Appendix A. However, as can be seen in the results of the test cases discussed in section 4, the accuracy of these methods may drop below their formal accuracy even though the test cases have $\mathcal{C}_{\infty}$ solutions and source functions. One important aspect that may be overlooked by the local analysis is the continuity between elements, or some equivalent property for finite differences. The symmetric scheme by Günter et al. [21] shows anisotropy independent results in case the diffusion tensor components are captured exactly by the staggered grid points. 


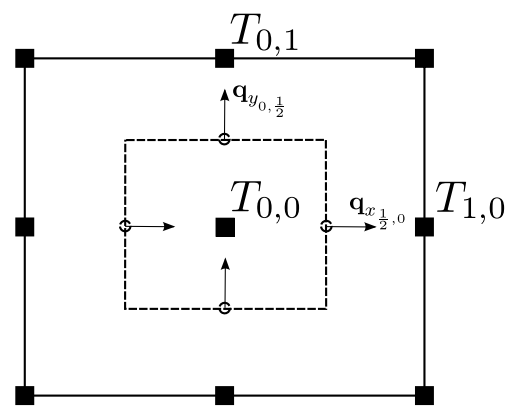

(a) Asymmetric

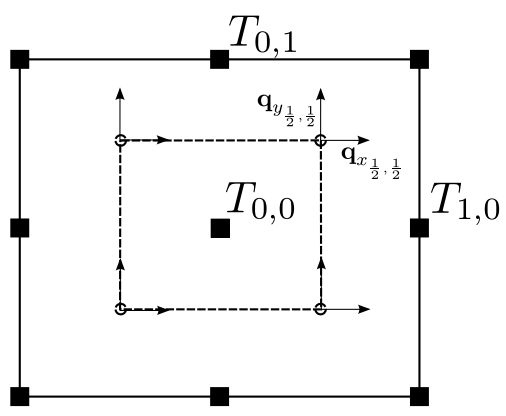

(b) Symmetric

Fig. 2. Control volumes, volume contour $S$ indicated by dashed line.

The symmetric scheme has $\mathcal{C}_{0}$ flux values and $\mathcal{C}_{1}$ temperature values at the flux points, in all directions, i.e. on the dual grid the solution is $\mathcal{C}_{1}$. Or speaking in finite-volume terms, the values of the solution on the flux points that connect neighbouring control volumes are $\mathcal{C}_{1}$. The equivalent control volume of the asymmetric scheme, which shows a loss of accuracy and convergence, is only $\mathcal{C}_{1}$ connected to the control volumes to the left/right and bottom/top, there is no direct connection with the diagonally neighbouring control volumes. To test the hypothesis that continuity between elements is important for the capturing of anisotropic diffusion we adapt the asymmetric finite-volume scheme so that all control volumes are $\mathcal{C}_{1}$ connected. Both the symmetric and the asymmetric scheme have equivalent finite-volume schemes, the difference now lies in the position of the fluxes on the surface of the control volume, see Fig. 2. A basic assumption of the finite-volume method is that the solution is spatially constant inside the control volumes. For the diffusion equation

$$
T_{t}=-\frac{1}{V} \oint_{S} \mathbf{q} \cdot \mathbf{n} d S+\frac{1}{V} \int_{V} f d V, \quad \mathbf{q}=-\mathbf{D} \cdot \nabla T,
$$

we get the same discrete formulation when using finite differences or finite volumes in case of equidistant grids, to be considered here. The mesh sizes are given by $\Delta x=\Delta y=h$. First we discuss the reference methods, namely the asymmetric finite-volume scheme and the symmetric finite-volume scheme.

\subsection{Asymmetric finite volume}

The first scheme we describe has the flux points defined on the cell-face centers, see Fig. 2(a). We have the following approximations for the gradients on the axes

$$
\begin{aligned}
& \left.\frac{\partial T}{\partial x}\right|_{i+\frac{1}{2}, j}=\frac{T_{i+1, j}-T_{i, j}}{h}, \\
& \left.\frac{\partial T}{\partial y}\right|_{i+\frac{1}{2}, j}=\frac{T_{i+1, j+1}+T_{i, j+1}-T_{i, j-1}-T_{i+1, j-1}}{4 h}, \\
& \left.\frac{\partial T}{\partial x}\right|_{i, j+\frac{1}{2}}=\frac{T_{i+1, j+1}+T_{i+1, j}-T_{i-1, j+1}-T_{i-1, j}}{4 h}, \\
& \left.\frac{\partial T}{\partial y}\right|_{i, j+\frac{1}{2}}=\frac{T_{i, j+1}-T_{i, j}}{h} .
\end{aligned}
$$

For the heat-conduction term we have

$$
\begin{aligned}
& \mathbf{q}_{i \pm \frac{1}{2}, j}=-\mathbf{D}_{i \pm \frac{1}{2}, j} \cdot\left(\left.\frac{\partial T}{\partial x}\right|_{i \pm \frac{1}{2}, j},\left.\frac{\partial T}{\partial y}\right|_{i \pm \frac{1}{2}, j}\right)^{T}, \\
& \mathbf{q}_{i, j \pm \frac{1}{2}}=-\mathbf{D}_{i, j \pm \frac{1}{2}} \cdot\left(\left.\frac{\partial T}{\partial x}\right|_{i, j \pm \frac{1}{2}},\left.\frac{\partial T}{\partial y}\right|_{i, j \pm \frac{1}{2}}\right)^{T} .
\end{aligned}
$$

Finally, the contour integral of the fluxes is approximated by

$$
\oint_{S} \mathbf{q} \cdot \mathbf{n} d S=\left[\left(\mathbf{q}_{i, j+\frac{1}{2}}-\mathbf{q}_{i, j-\frac{1}{2}}\right) \cdot(0,1)+\left(\mathbf{q}_{i+\frac{1}{2}, j}-\mathbf{q}_{i-\frac{1}{2}, j}\right) \cdot(1,0)\right] h .
$$




\subsection{Symmetric finite volume}

Another approach is taken by Günter et al. [21], they use a symmetric scheme (with a symmetric linear operator) that is mimetic by maintaining the self-adjointness of the differential operator. The flux points are placed at the vertices of the control volumes. The control volume with the location of the fluxes is given in Fig. 2(b). The divergence terms are determined at the center points in the following manner

$$
\begin{aligned}
& \left.\frac{\partial T}{\partial x}\right|_{i+\frac{1}{2}, j+\frac{1}{2}}=\frac{T_{i+1, j+1}+T_{i+1, j}-T_{i, j+1}-T_{i, j}}{2 h}, \\
& \left.\frac{\partial T}{\partial y}\right|_{i+\frac{1}{2}, j+\frac{1}{2}}=\frac{T_{i, j+1}+T_{i+1, j+1}-T_{i+1, j}-T_{i, j}}{2 h} .
\end{aligned}
$$

Next, the diffusion tensor is applied to obtain the heat flux

$$
\begin{aligned}
& \mathbf{q}_{i \pm \frac{1}{2}, j \pm \frac{1}{2}}=-\mathbf{D}_{i \pm \frac{1}{2}, j \pm \frac{1}{2}} \cdot\left(\left.\frac{\partial T}{\partial x}\right|_{i \pm \frac{1}{2}, j \pm \frac{1}{2}},\left.\frac{\partial T}{\partial y}\right|_{i \pm \frac{1}{2}, j \pm \frac{1}{2}}\right)^{T}, \\
& \mathbf{q}_{i \pm \frac{1}{2}, j \mp \frac{1}{2}}=-\mathbf{D}_{i \pm \frac{1}{2}, j \mp \frac{1}{2}} \cdot\left(\left.\frac{\partial T}{\partial x}\right|_{i \pm \frac{1}{2}, j \mp \frac{1}{2}},\left.\frac{\partial T}{\partial y}\right|_{i \pm \frac{1}{2}, j \mp \frac{1}{2}}\right)^{T} .
\end{aligned}
$$

Finally, the contour integral of the fluxes is approximated by

$$
\begin{aligned}
\oint_{S} \mathbf{q} \cdot \mathbf{n} d S= & \frac{1}{2}\left[\left(\mathbf{q}_{i+\frac{1}{2}, j+\frac{1}{2}}+\mathbf{q}_{i+\frac{1}{2}, j-\frac{1}{2}}\right) \cdot(1,0)-\left(\mathbf{q}_{i-\frac{1}{2}, j-\frac{1}{2}}+\mathbf{q}_{i+\frac{1}{2}, j-\frac{1}{2}}\right) \cdot(0,1)\right. \\
& \left.+\left(\mathbf{q}_{i+\frac{1}{2}, j+\frac{1}{2}}+\mathbf{q}_{i-\frac{1}{2}, j+\frac{1}{2}}\right) \cdot(0,1)-\left(\mathbf{q}_{i-\frac{1}{2}, j-\frac{1}{2}}+\mathbf{q}_{i-\frac{1}{2}, j+\frac{1}{2}}\right) \cdot(1,0)\right] h .
\end{aligned}
$$

\subsection{Eight point flux scheme}

To investigate the importance of connectivity we apply a cell-face-centered flux (CF) scheme and a vertex-centered flux (VF) scheme on a grid with varying connectivity. The grid is varied through a parameter $e, e \in[0, \sqrt{2} h]$, where for $e=0$ and $e=\sqrt{2} h$ the grid is uniform and rectangular, and for $e$ between 0 and $\sqrt{2} h$ we have a hybrid grid consisting of octagons with edges of lengths $e$ and $\Delta s$ and squares with edge length $\Delta s$ (Fig. 3). Due to the geometric constraints imposed by the combination of octagons and quadrilaterals, the grid structure can only vary through the stepsize $h$ and the parameter $e$, and is thus structured by definition.

Note that it holds $\Delta s=h-e / \sqrt{2}$, and hence, with all edges of the same length, $e=\Delta s, \Delta s=(2-\sqrt{2}) h$. The latter situation has been depicted in Fig. 4(a). The two extreme cases $e=0$ and $e=\sqrt{2} h$ are given in Figs. 4(b) and 4(c), respectively. Note that in the case of $e=\sqrt{2} h$ the grid may be better aligned to features that are oblique with respect to the coordinate axes, but it will be coarser and have less finite volumes. For each volume we approximate the contour integral of the fluxes as follows

$$
\oint_{S} \mathbf{q} \cdot \mathbf{n} d S \approx \sum_{i=1}^{M} \tilde{\mathbf{q}}_{i} \cdot \mathbf{n}_{i} l_{i}
$$

where $\tilde{\mathbf{q}}_{i}$ is an approximation of the flux across cell face $i$, and where $M=4$ and $M=8$ for the square and octagonal finite volume, respectively. Extending this approach to three dimensions is straightforward for a uniform Cartesian grid. For $e \in\langle 0, \sqrt{2} h\rangle$ it is not possible to apply a grid with varying values for $h$.

\subsubsection{Vertex-centered fluxes}

The VF scheme has the following description of the divergence

$$
\text { octagonal volume: } \begin{aligned}
\oint_{S} \mathbf{q} \cdot \mathbf{n} d S= & \frac{1}{2} \sqrt{2}\left[\left(\overline{\mathbf{q}}_{u r, r u}-\overline{\mathbf{q}}_{l d, d l}\right) \cdot(1,1)+\left(\overline{\mathbf{q}}_{r d, d r}-\overline{\mathbf{q}}_{u l, l u}\right) \cdot(1,-1)\right] e \\
& +\left[\left(\overline{\mathbf{q}}_{r u, r d}-\overline{\mathbf{q}}_{l u, l d}\right) \cdot(1,0)+\left(\overline{\mathbf{q}}_{u l, u r}-\overline{\mathbf{q}}_{d l, d r}\right) \cdot(0,1)\right] \Delta s,
\end{aligned}
$$

Square volume: $\quad \oint_{S} \mathbf{q} \cdot \mathbf{n} d S=\left[\left(\overline{\mathbf{q}}_{r u, r d}-\overline{\mathbf{q}}_{l u, l d}\right) \cdot(1,0)+\left(\overline{\mathbf{q}}_{r u, l u}-\overline{\mathbf{q}}_{r d, l d}\right) \cdot(0,1)\right] \Delta s$,

where we have to retain the normality of the resulting unit direction vector for the averaging of the fluxes. 


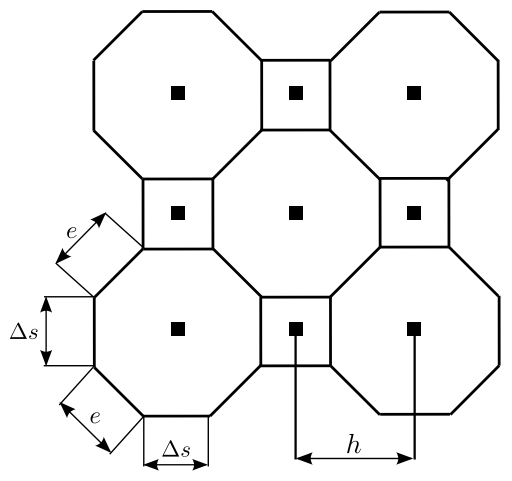

Fig. 3. Part of hybrid grid $(0<e<\sqrt{2} h)$.

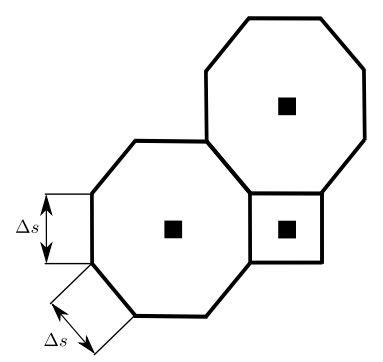

(a)

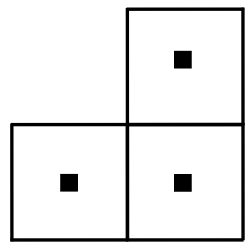

(b)

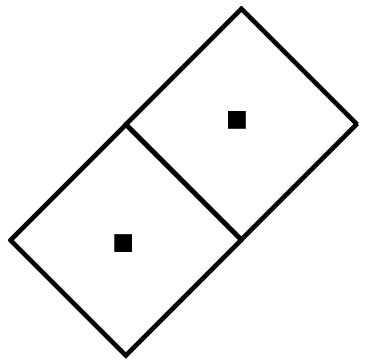

(c)

Fig. 4. (a) $e=\Delta s$, (b) $e=0$, (c) $e=\sqrt{2} h$.

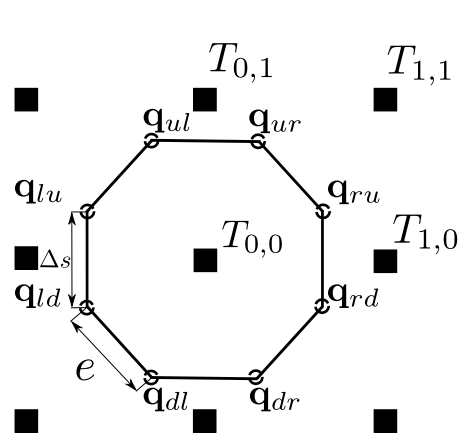

(a) octagonal volume
$T_{-1,1} \quad T_{1,1}$

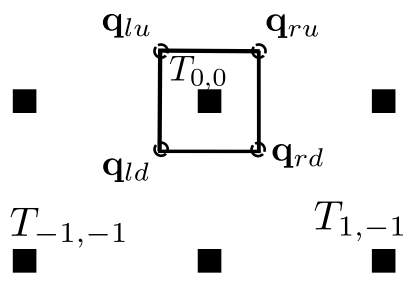

(b) Square volume

Fig. 5. Control volumes with vertex centered fluxes, contour $S$ indicated by solid line.

The averaged flux vectors $\overline{\mathbf{q}}_{u r, r u}, \overline{\mathbf{q}}_{u l, l u}, \cdots$ generally denoted as $\overline{\mathbf{q}}_{A, B}$, are computed as

$$
\overline{\mathbf{q}}_{A, B}=\frac{1}{4}\left[\left(\left(D_{\|}\right)_{A}+\left(D_{\|}\right)_{B}-\left(D_{\perp}\right)_{A}-\left(D_{\perp}\right)_{B}\right)\left(\mathbf{b b}^{T}\right)_{A, B}+\left(\left(D_{\perp}\right)_{A}+\left(D_{\perp}\right)_{B}\right) \mathcal{I}\right]\left(\nabla T_{A}+\nabla T_{B}\right),
$$

with $\mathbf{b}_{A, B}=\left(\mathbf{b}_{A}+\mathbf{b}_{B}\right) /\left|\mathbf{b}_{A}+\mathbf{b}_{\mathbf{B}}\right|$. Here the gradient approximations follow from the interpolation, see Fig. 5 for the nomenclature. Note that applying a separate normalized averaging of the unit direction vectors gives a division by zero if the unit direction vectors are opposed. In this case we simply have to pick one of the directions, or, if we know that an opposed direction means there is an $\mathrm{O}$-point, we can set the respective parallel flux to zero. Almost directly opposed field lines are numerically not an issue for the normalization. With $O$-point we refer to the center of rotation of closed field lines, exactly at the $O$-point the direction is not defined.

In case of diffusion tensor values not defined exactly in the flux points but rather in the cells, or in case of sharply varying densities, harmonic averaging is preferred for the tensor values on the cell faces (see Edwards and Rogers [13] and Sharma and Hammett [33]). This is generally not the case, e.g. for MHD simulations of fusion plasmas where the density 


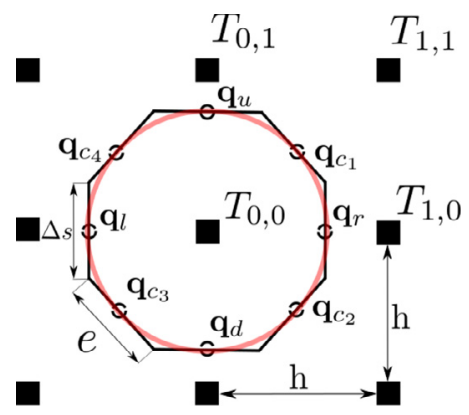

(a) octagonal volume

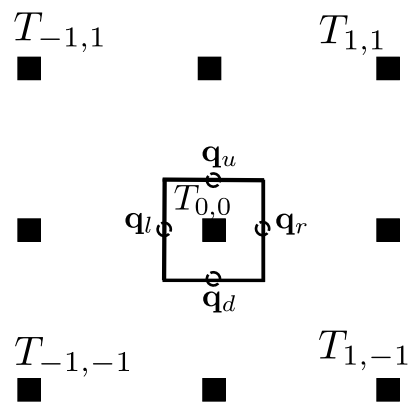

(b) Square volume

Fig. 6. Control volumes with cell-face centered fluxes, contour $S$ indicated by solid line.

and the diffusion tensor vary smoothly. For this reason we do not consider piecewise constant diffusion tensors, rather we apply a continuous nine-point interpolation. We define the diffusion tensor values exactly in the flux evaluation points.

\subsubsection{Cell-face centered fluxes}

With the flux vectors in the corner points of the control volume pointing in a direction of $45^{\circ}$ with respect to the coordinate axes, the scheme is written as

$$
\text { octagonal volume: } \begin{aligned}
\oint_{S} \mathbf{q} \cdot \mathbf{n} d S= & {\left[\frac{1}{2} \sqrt{2}\left(\left(\mathbf{q}_{c_{1}}-\mathbf{q}_{c_{3}}\right) \cdot(1,1)+\left(\mathbf{q}_{c_{2}}-\mathbf{q}_{c_{4}}\right) \cdot(1,-1)\right) e\right.} \\
& \left.+\left(\left(\mathbf{q}_{r}-\mathbf{q}_{l}\right) \cdot(1,0)+\left(\mathbf{q}_{u}-\mathbf{q}_{d}\right) \cdot(0,1)\right) \Delta s\right],
\end{aligned}
$$

Square volume: $\quad \oint_{S} \mathbf{q} \cdot \mathbf{n} d S=\left[\left(\mathbf{q}_{r}-\mathbf{q}_{l}\right) \cdot(1,0)+\left(\mathbf{q}_{u}-\mathbf{q}_{d}\right) \cdot(0,1)\right] \Delta s$,

where the cell-face fluxes $\mathbf{q}_{c_{1}}, \mathbf{q}_{c_{2}}, \mathbf{q}_{c_{3}}, \mathbf{q}_{c_{4}}$ and $\mathbf{q}_{r}, \mathbf{q}_{l}, \mathbf{q}_{u}, \mathbf{q}_{d}$ are given in Fig. 6. Using a circle with radius $\frac{1}{2} \sqrt{2} h$ we ensure that the flux points $c_{1}, c_{2}, c_{3}, c_{4}$ lie exactly on the average points of the four surrounding nodes (see Fig. 6). For the $x$ and $y$-derivatives in the vertical and horizontal flux points respectively we use a nine-point interpolation scheme for the temperature, see section 3. For $e=0$ the scheme reduces to the asymmetric scheme from section 2.1. For $e=\sqrt{2} h$ we end up with a symmetric description for the derivatives. This is equivalent to the symmetric scheme from section 2.2 if we use symmetric approximations for the derivatives. For verification we will also show the results for the asymmetric and symmetric schemes from sections 2.1 and 2.2 respectively.

\section{Interpolation for fluxes}

Given the formulation of the discrete divergence for the VF and CF scheme we need to find the discrete description of the fluxes. We discuss the use of a local and a non-local flux approximation.

\subsection{Local flux approximation for eight point flux scheme}

Suppose we apply a local flux interpolation with the volumes surrounding the flux point, assuming we have cell-centered temperature values. The flux through the interfaces is determined using subcell-descriptions of the temperature. Here triangles may be formed by connecting the center points of three neighbouring volumes, see Fig. 7 . We need two triangles for the determination of one edge flux, as per triangle we determine half of the flux going through the edge (see Fig. 8). We consider the approach described in e.g. Aavatsmark et al. [4,5] and Edwards and Rogers [13], i.e. we consider subcells with one temperature value per subcell face. We have already surrounded each vertex with an interaction triangle, which connects the surrounding temperature unknowns. The octagonal volume has eight vertices and thus eight interaction triangles, the square volume has four. It is clear that using a mixture of octagons and squares for the finite volumes leads to a mixture of nine-point stencils and five-point stencils when applying a locally conservative method (see Fig. 8). Only for $e=\sqrt{2} h$ and $e=0$ a nine-point stencil emerges. It is known from literature that to properly resolve non-grid-aligned anisotropic diffusion with a linear scheme at least a nine-point stencil is required (see e.g. Umansky et al. [36]). The five-point stencil for the square volumes, which is unavoidable when using a combination of octagonal and square volumes, does not satisfy this requirement. Hence we will not further consider this multi-point flux approach. This non-locality is a limitation of the eight point flux scheme. 


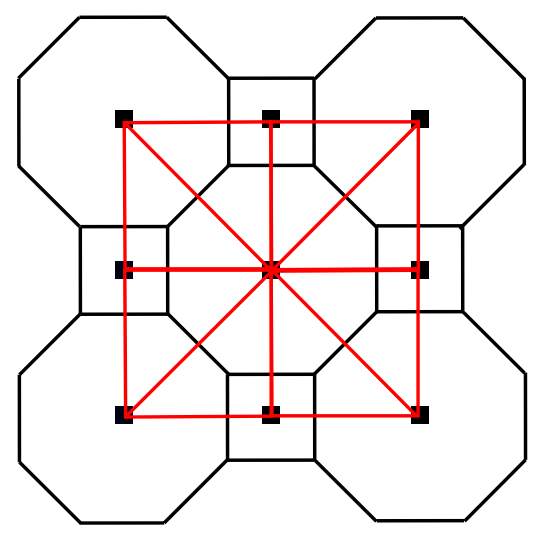

Fig. 7. Interaction triangles, indicated by red lines. (For interpretation of the references to color in this figure legend, the reader is referred to the web version of this article.)

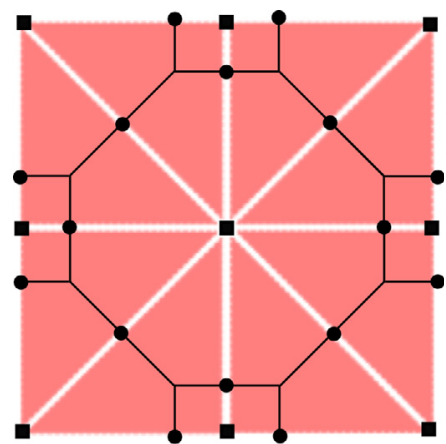

(a)

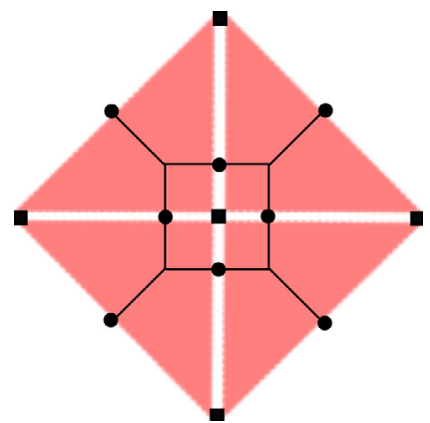

(b)

Fig. 8. Interaction triangles for (a) octagonal volume, (b) square volume, solid squares ( $\square$ ) indicate temperature unknowns and solid circles ( $\bullet$ ) indicate added unknowns to solve for the fluxes.

\subsection{Non-local flux approximation}

Since the flux points $r, l, u, d$ are off-center with respect to the centered temperature values we are forced to involve more points to obtain second-order accuracy in approximating the derivatives in these flux points. To have second-order accuracy for general values of $e$ we apply biquadratic interpolation of the surrounding temperature values. When applying a nine-point interpolation we are assuming to have a continuous temperature and flux distribution. In case of a smoothly varying diffusion tensor and temperature value this assumption is realistic. Also see the Center Flux Scheme by Ferziger and Perić [18].

We have the following description for the derivatives:

$$
\begin{aligned}
& \left.\frac{\partial T}{\partial x}\right|_{x, y} \approx F(x, y)=\frac{\partial}{\partial x} \sum_{i=0}^{2} \sum_{j=0}^{2} \gamma_{i j} x^{i} y^{j}, \\
& \left.\frac{\partial T}{\partial y}\right|_{x, y} \approx G(x, y)=\frac{\partial}{\partial y} \sum_{i=0}^{2} \sum_{j=0}^{2} \gamma_{i j} x^{i} y^{j},
\end{aligned}
$$

where the set of coefficients $\gamma_{i j}$ is found by applying the Vandermonde matrix to the cell-centered temperature unknowns and subsequently differentiating the interpolation function, see Appendices B and C. A second option is to weigh the symmetric approximation of the derivatives given e.g. by equation (2) such that the derivatives are given by

$$
\begin{aligned}
T_{x} \approx & \alpha_{r u} \frac{T_{i+1, j+1}+T_{i+1, j}-T_{i, j+1}-T_{i, j}}{2 h}+\alpha_{l u} \frac{T_{i, j}+T_{i, j+1}-T_{i-1, j+1}-T_{i-1, j}}{2 h} \\
& +\alpha_{l d} \frac{T_{i, j}+T_{i, j-1}-T_{i-1, j}-T_{i-1, j-1}}{2 h}+\alpha_{r d} \frac{T_{i+1, j}+T_{i+1, j-1}-T_{i, j}-T_{i, j-1}}{2 h},
\end{aligned}
$$




$$
\begin{aligned}
T_{y} \approx & \alpha_{r u} \frac{T_{i+1, j+1}+T_{i, j+1}-T_{i, j}-T_{i+1, j}}{2 h}+\alpha_{l u} \frac{T_{i, j+1}+T_{i-1, j+1}-T_{i-1, j}-T_{i, j}}{2 h} \\
& +\alpha_{l d} \frac{T_{i, j}+T_{i-1, j}-T_{i-1, j-1}-T_{i, j-1}}{2 h}+\alpha_{r d} \frac{T_{i+1, j}+T_{i, j}-T_{i, j-1}-T_{i+1, j-1}}{2 h} .
\end{aligned}
$$

These derivatives are consistently summed in a weighted fashion, where the weights are given by

$$
\begin{aligned}
\alpha_{r u} & =\left(\frac{1}{2}+\frac{x}{h}\right)\left(\frac{1}{2}+\frac{y}{h}\right), \\
\alpha_{l u} & =\left(\frac{1}{2}-\frac{x}{h}\right)\left(\frac{1}{2}+\frac{y}{h}\right), \\
\alpha_{r d} & =\left(\frac{1}{2}+\frac{x}{h}\right)\left(\frac{1}{2}-\frac{y}{h}\right), \\
\alpha_{l d} & =\left(\frac{1}{2}-\frac{x}{h}\right)\left(\frac{1}{2}-\frac{y}{h}\right) .
\end{aligned}
$$

We call the resulting coefficients locally symmetric, see Appendix C for the contribution to the linear operator.

\section{Numerical results and methodological adaptations}

In the following test cases we will consider both Vandermonde and locally symmetric coefficients. If results are similar we only show the results for the Vandermonde coefficients. The test case descriptions are given by (8), (9), (10), (11), (12) and (13). Dirichlet boundary conditions were used for all test cases. The diffusion equation is approximated on a uniform Cartesian grid with $\Delta x=\Delta y=h$.

\subsection{Closed field-line test cases}

In this section we show the results for closed field-line test cases with extreme levels of anisotropy. In this test case b $\nabla T=0$. We vary the values of the parameters $e$ and $\zeta$. The test cases we consider have continuous distributions for the diffusion tensor and the temperature. The error norm is defined by

$$
\epsilon_{\infty}=\frac{|\tilde{T}-T|_{\max }}{|T|_{\max }}
$$

where $\tilde{T}$ is the approximate temperature. The problem is considered on a square domain, described by $-0.5 \leq x, y \leq 0.5$. The following steady-state solution is assumed on the domain:

$$
T(x, y)=1-\left(x^{2}+y^{2}\right)^{3 / 2}, \quad x, y \in[-0.5,0.5],
$$

with the unit direction vector given by

$$
\mathbf{b}=\frac{1}{\sqrt{x^{2}+y^{2}}}\left(\begin{array}{c}
-y \\
x
\end{array}\right) .
$$

In Fig. 9, we study the accuracy of the various schemes for two anisotropic cases, one being extremely anisotropic, $\varsigma=$ $10^{9}$. The main observation to be made from Fig. 9 is that for the extremely anisotropic $\varsigma=10^{9}$ case only the symmetric scheme by Günter et al. and the CF scheme, with $e=\sqrt{2} h$ and locally symmetric coefficients, are second-order convergent and moreover of low error magnitude. Using the locally symmetric coefficients; for $e \rightarrow \sqrt{2} h$ we go from a non-local interpolation involving all the temperature unknowns for each derivative to a local interpolation involving only the volumes surrounding the flux points. This explains the jump in (order of) accuracy going from $e=0.95 \sqrt{2} h$ to $e=\sqrt{2} h$ in Fig. 9(b). In Fig. 10 we still present results obtained with the VF scheme for the same test case. Here, as opposed to the CF scheme with locally symmetric coefficients (Fig. 9), the VF scheme with locally symmetric coefficients does not perform well for $\varsigma=10^{9}$.

\section{2. e-Dependency}

We proceed by studying the $e$-dependency in more detail. We consider a constant angle test case which is described by

$$
T(x, y)=x y[\sin (\pi x) \sin (\pi y)]^{10}, \quad x, y \in[0,1],
$$

where the angle of misalignment $\alpha$ is set to a constant value. The solution simulates a temperature peak. For both the cell face-centered scheme and the vertex-centered scheme we observe a noticeable influence of the dominant angle of anisotropy on the $e$-dependency of the error, see Fig. 11. Concerning the effect of $e$-variation, notice the opposite behavior 


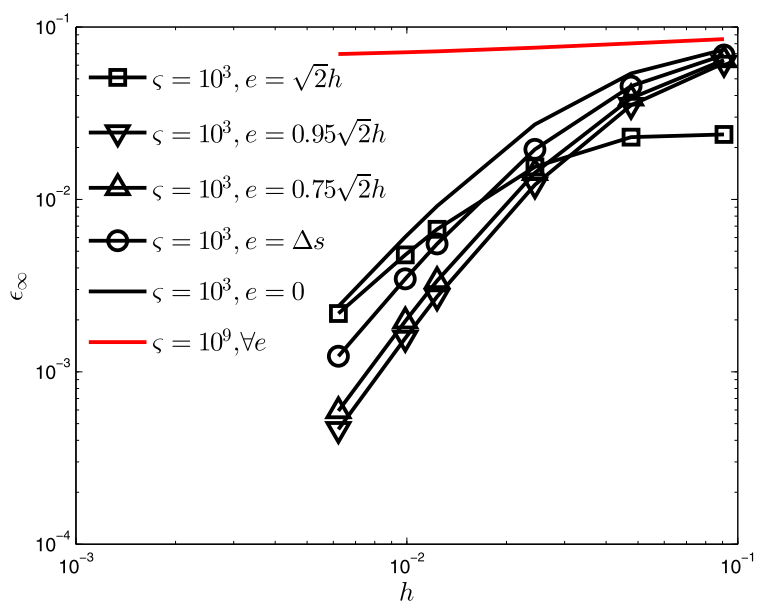

(a)

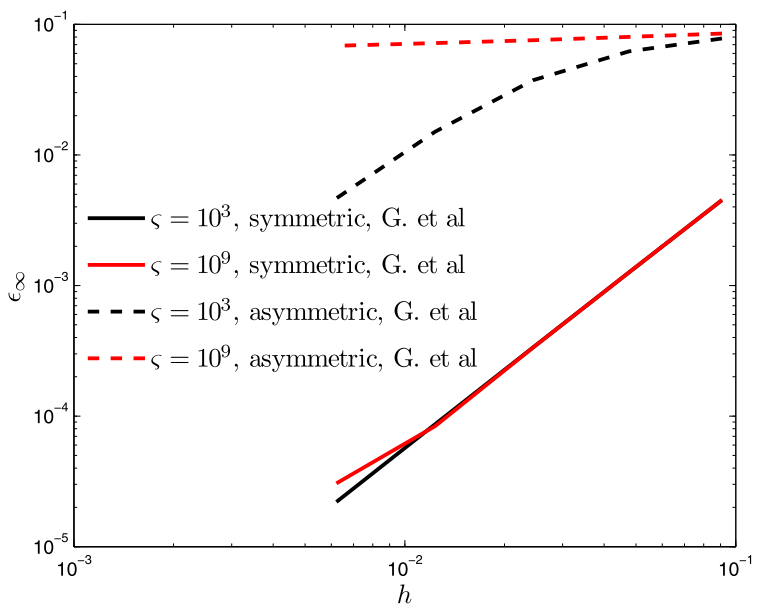

(c)

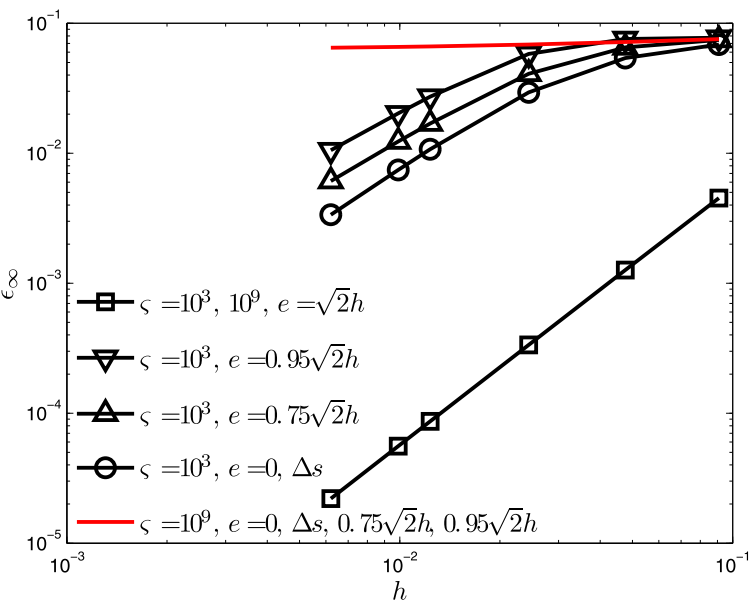

(b)

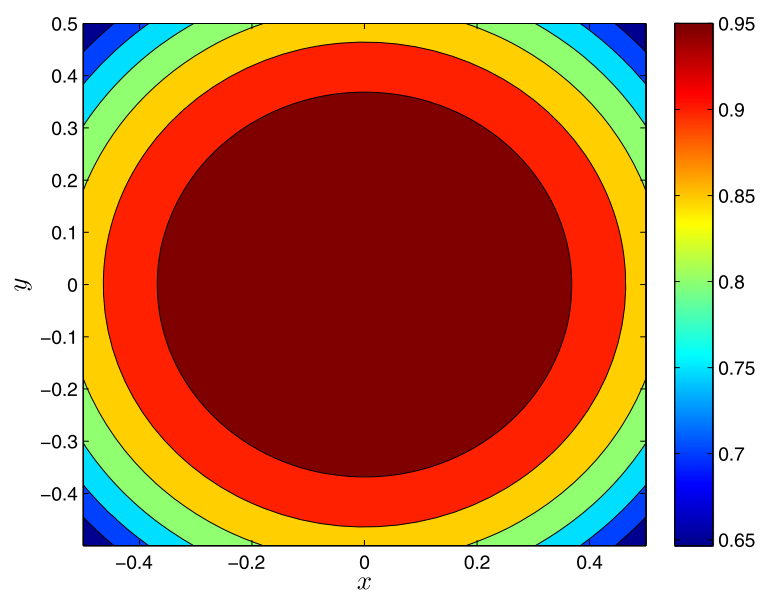

(d)

Fig. 9. Test case (8), error $\epsilon_{\infty}$ for anisotropy ratios $\varsigma=10^{3}$ and $\varsigma=10^{9}$, CF scheme with (a) Vandermonde coefficients, (b) locally symmetric coefficients, and (c) the asymmetric and symmetric scheme of Günter et al., (d) solution contours. (For interpretation of the colors in this figure, the reader is referred to the web version of this article.)

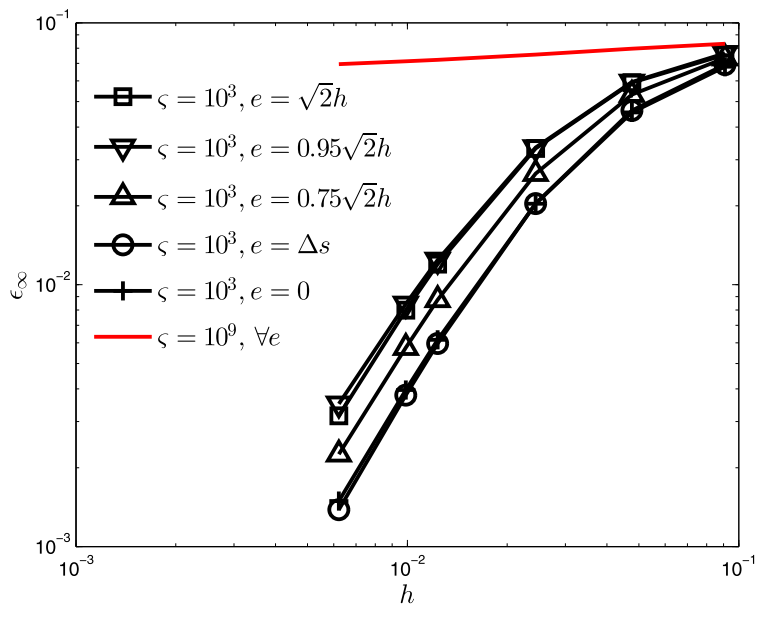

(a)

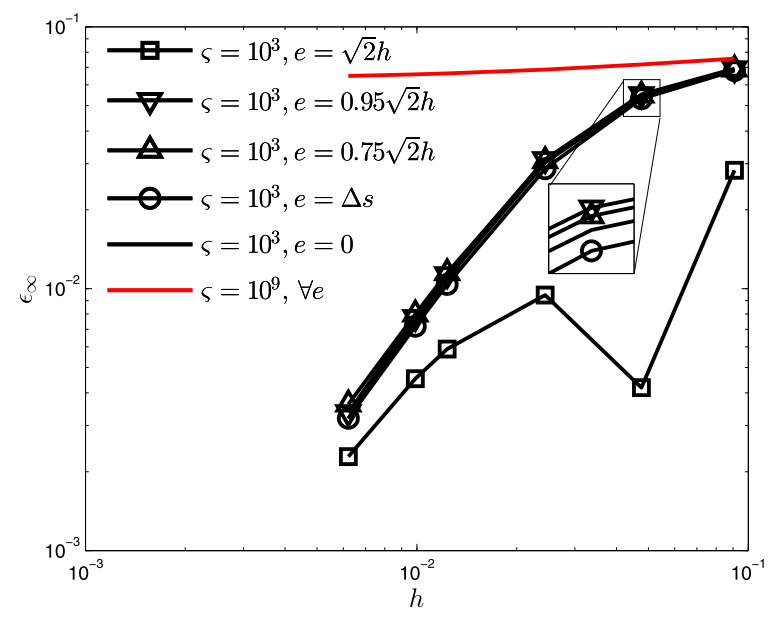

(b)

Fig. 10. Test case (8), error $\epsilon_{\infty}$ for anisotropy ratios $\varsigma=10^{3}$ and $\varsigma=10^{9}$, VF scheme with (a) Vandermonde coefficients, (b) locally symmetric coefficients. 


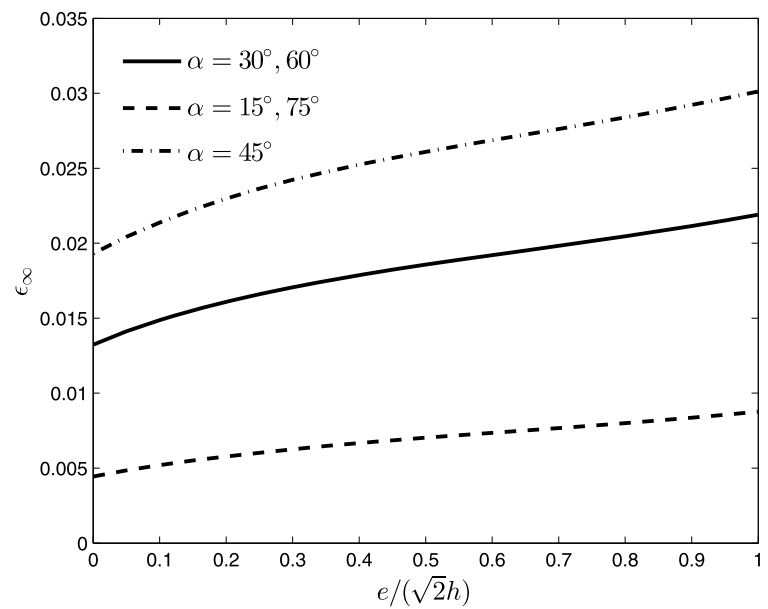

(a)

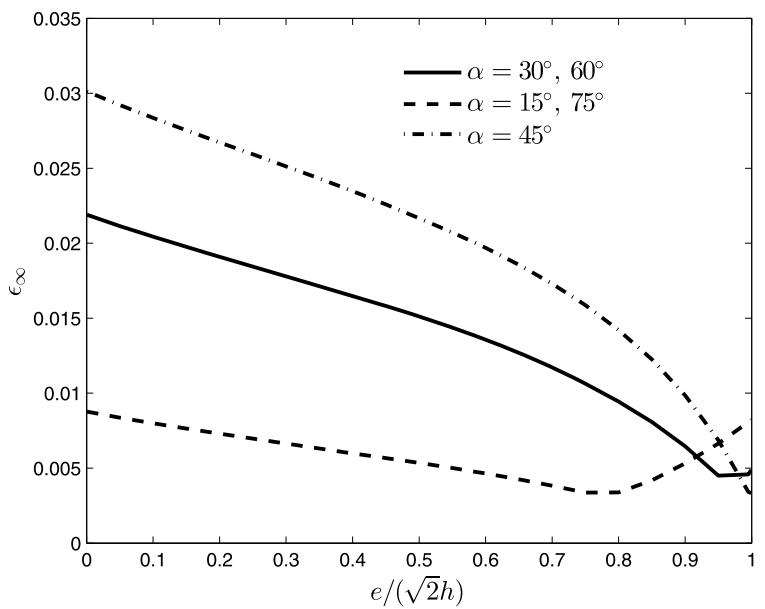

(b)

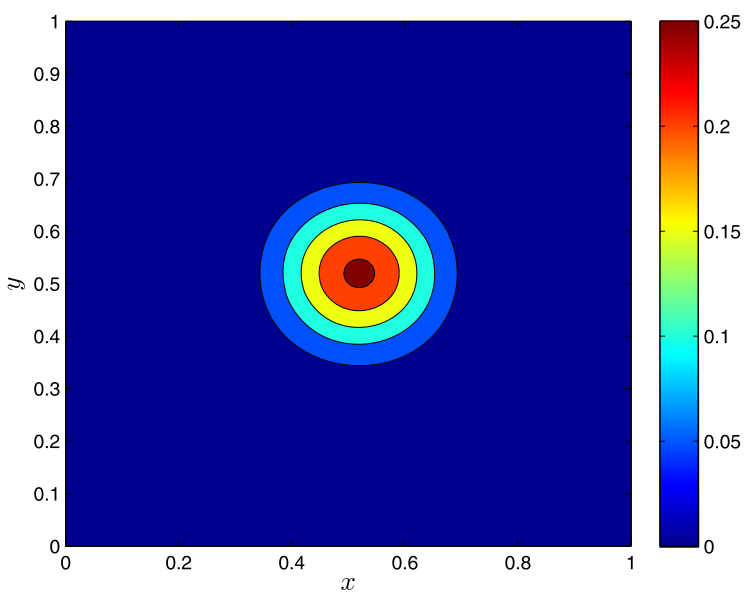

(c)

Fig. 11. Test case (9), error $\epsilon_{\infty}$ for $\varsigma=10^{9}$, a $50 \times 50$ grid and varying values of $e$, (a) VF scheme, (b) CF scheme, (c) solution contours. (For interpretation of the colors in this figure, the reader is referred to the web version of this article.)

of the VF scheme and the CF scheme. Also note that for most values of $e$ the error increases as the dominant angle of anisotropy goes to $45^{\circ}$. At and near $e=\sqrt{2} h$, for the CF scheme the behavior is opposite though; CF scheme's best results are obtained for $\alpha=45^{\circ}$. For the CF scheme, we also clearly observe the best behavior is at or near $e=\sqrt{2} h$.

\subsection{Adaptations for closed field lines}

There is a clear distinction between closed and open field-line cases. The closed field-line cases allow for specific adaptations to improve the accuracy. The common point around which the field lines rotate is called the 0 -point.

\subsubsection{Topology mimicking}

To incorporate the closed field-line topology in the finite-volume discretization we enforce that the unit direction vectors are tangential to the edges of the finite volume, see Fig. 12. Also note that in this way we enforce that $\nabla \cdot \mathbf{b}=0$. In general this is not consistent with the diffusion equation because in general $\nabla \cdot \mathbf{b} \neq 0$. Sovinec et al. [35] devised a test containing only closed field lines to directly compare the perpendicular numerical diffusion to the actual numerical diffusion. The exact solution and the forcing function are given by

$$
T(x, y)=\frac{1}{D_{\perp}} \psi, \quad f(x, y)=2 \pi^{2} \psi, \quad \psi(x, y)=\cos (\pi x) \cos (\pi y), \quad x, y \in[-0.5,0.5] .
$$

The error in the perpendicular diffusion is given by $\left|T(0,0)^{-1}-D_{\perp}\right|$. We use homogeneous Dirichlet boundary conditions. The field lines are tangential to the contours of constant temperature, i.e. 


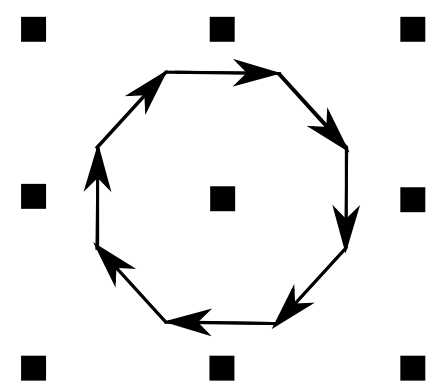

(a)

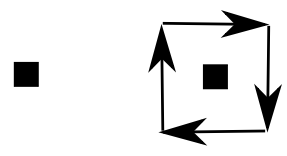

(b)

Fig. 12. Topology local field lines for (a) octagonal volume, (b) quadrilateral volume.

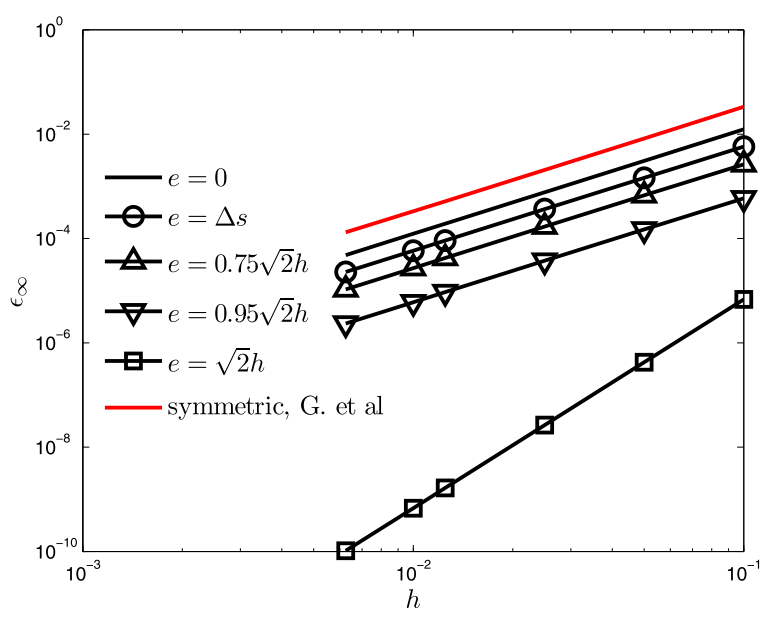

(a)

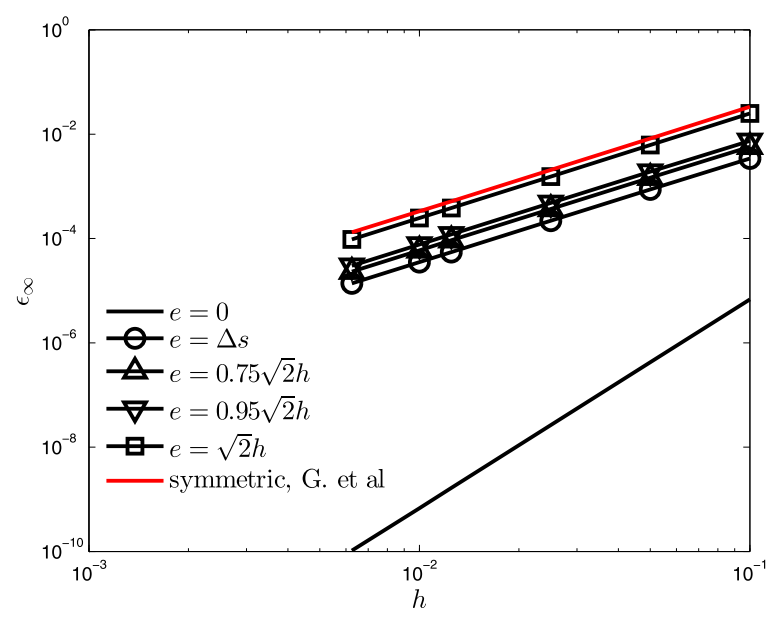

(b)

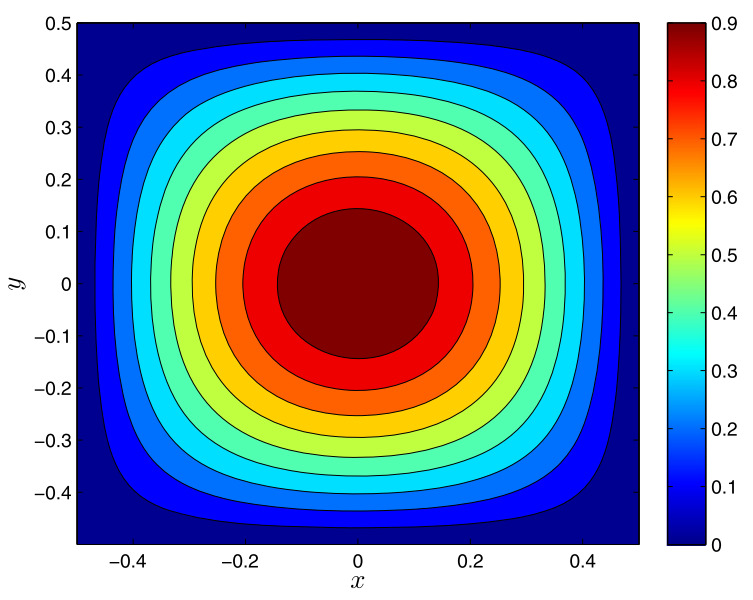

(c)

Fig. 13. Test case (10), error $\epsilon_{\infty}, \varsigma=10^{9}$, with varying values of $e$, using Vandermonde coefficients. Here the symmetric scheme by Günter et al. is used as a reference. (a) VF scheme, (b) CF scheme, (c) solution contours. (For interpretation of the colors in this figure, the reader is referred to the web version of this article.)

$$
\mathbf{b}=\frac{1}{\sqrt{\psi_{x}^{2}+\psi_{y}^{2}}}\left(\begin{array}{c}
-\psi_{y} \\
\psi_{x}
\end{array}\right) .
$$

We obtain fourth-order convergence for the Sovinec test case independent of the level of anisotropy, using Vandermonde coefficients, with $e=\sqrt{2} h$, with vertex averaged gradients and with the unit direction vectors enforced to be tangent to the cell faces, see Fig. 13(a). The Sovinec test case has closed field lines, as such the topology of the entire domain is mimicked 


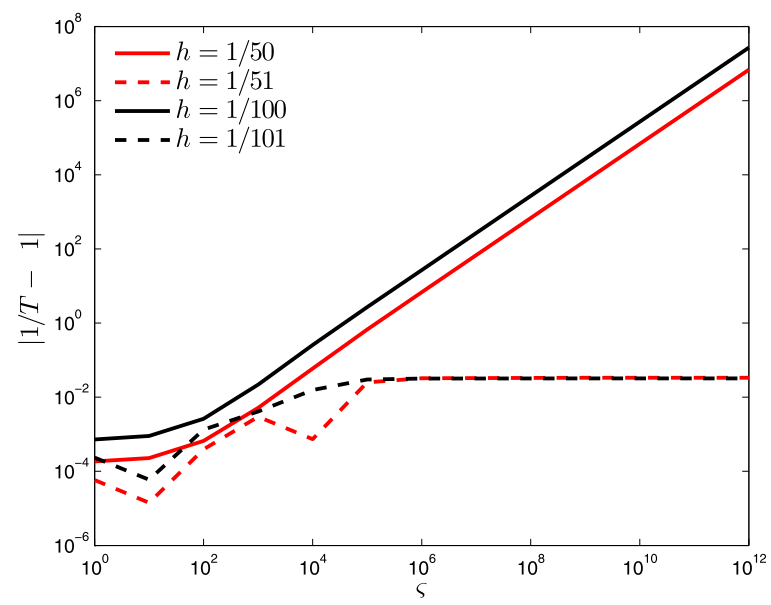

(a)

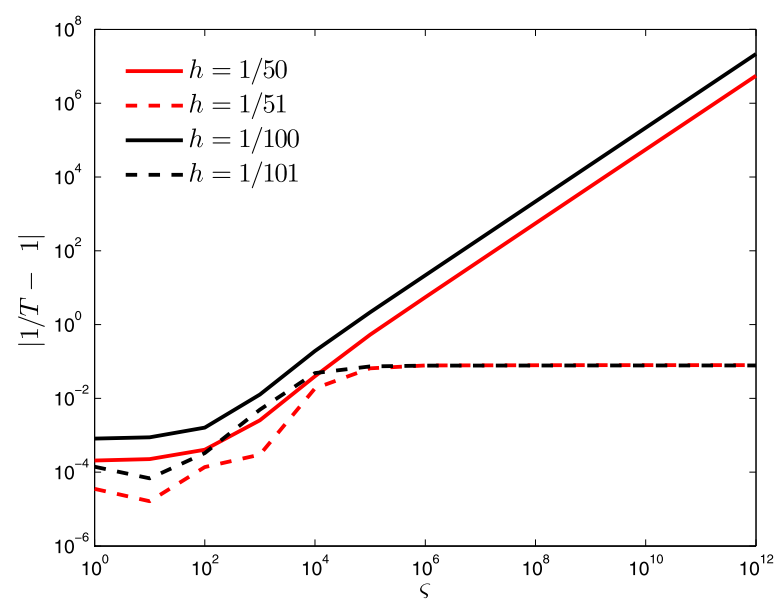

(b)

Fig. 14. Test case (10), error $\left|T^{-1}-1\right|$ with varying anisotropy, $e=\Delta s$, Vandermonde coefficients, with odd and even number of finite volumes (a) VF scheme, (b) CF scheme.

by the individual volumes. We believe this is key to the high-order approximation. We see that the accuracy increases monotonously as $e$ goes to $\sqrt{2} h$, and that it seems to jump to fourth-order for $e=\sqrt{2} h$. Further, we see the inverse for the CF scheme, where we have fourth-order accuracy for $e=0$ and second order for higher values of $e$, with a subsequent decrease in accuracy for increasing $e$, see Fig. 13(b).

The VF scheme with exact unit direction vectors and the VF scheme with averaged unit direction vectors are identical since the local values for the unit direction vector (and thus also its approximation) do not play a role in the accuracy. We note that using the locally symmetric coefficients the results are almost identical, without the fourth-order convergence for $e=\sqrt{2} h$.

If we force the field lines for the parallel diffusion coefficient to be tangential to the cell faces, letting the field lines for the perpendicular diffusion coefficient untouched, we get the same result as setting $D_{\|}$to zero everywhere in the domain for any level of anisotropy without the fourth-order accuracy for $e=\sqrt{2} h$.

\subsubsection{Importance of 0 -point}

We now apply the VF and CF scheme to the Sovinec test case, without the foregoing enforcement of field line topology. We notice a huge difference between odd and even numbered grids; both the VF and the CF scheme perform much better on odd-numbered grids than on even-numbered grids. On the odd-numbered grids, for both schemes, the error of the perpendicular diffusion remains fairly constant with increasing anisotropy, see Fig. 14 .

The gain in accuracy is caused by the fact that the central volume exactly mimicks the topology of the closed field line with $\mathbf{b} \cdot \nabla T=0$ leading to a zero contribution of $D_{\|}$. To verify this we enforce $D_{\|}=0$ for the flux points positioned closest to the $O$-point for the even-numbered grids. The results, given in Fig. 15, show that enforcing $D_{\|}=0$ close to the $O$-point has a similar effect as having a volume exactly on the $O$-point.

For both the VF scheme and the CF scheme we get $\mathcal{O}\left(h^{4}\right)$ convergence for $e \rightarrow \sqrt{2} h$ and $e \rightarrow 0$ respectively for the isotropic case if we have a finite volume exactly on the $O$-point, see Fig. 16(c). The 4th-order convergence becomes independent of the level of anisotropy if we mimick the topology everywhere in the domain as we saw in section 4.3 .

\subsubsection{Enforcing $D_{\|}=0$}

We see that enforcing $D_{\|}=0$ around the $O$-point, as well as placing a volume exactly on the $O$-point leads to a considerable gain in accuracy for the Sovinec test case. This approach is interesting as a means to improve existing methods because the location of the $O$-point can be derived from the magnetic field data. The same caveat as for the topology mimicking holds here; validity is limited to situations where the parallel flux is zero. However, it is not necessarily limited to steady cases. The involvement with the problem parameter $D_{\|}$might be seen as a form of model reduction. If a point on the last closed field line is found and subsequently $D_{\|}$is enforced to be zero on the line connecting the outer point and the $O$-point (see Fig. 17), we now have full second-order accuracy for $e=0, e=\Delta s$ and $e=\sqrt{2} h$, see Fig. 18 . We know from Van Es et al. [37] that the symmetric scheme by Günter et al. loses anisotropy independence for tilted elliptic temperature distributions. Note that the symmetric scheme is identical to the symmetric finite volume scheme for a uniform rectangular grid. This tilted elliptic distribution has no symmetry axes aligned with the coordinate axes and as such is more general than the previous test case.

The distribution for the tilted test case is given by

$$
T(x, y)=1-\left(a^{2}(x \cos \theta+y \sin \theta)^{2}+b^{2}(x \sin \theta-y \cos \theta)^{2}\right)^{3 / 2}, \quad x, y \in[-0.5,0.5],
$$




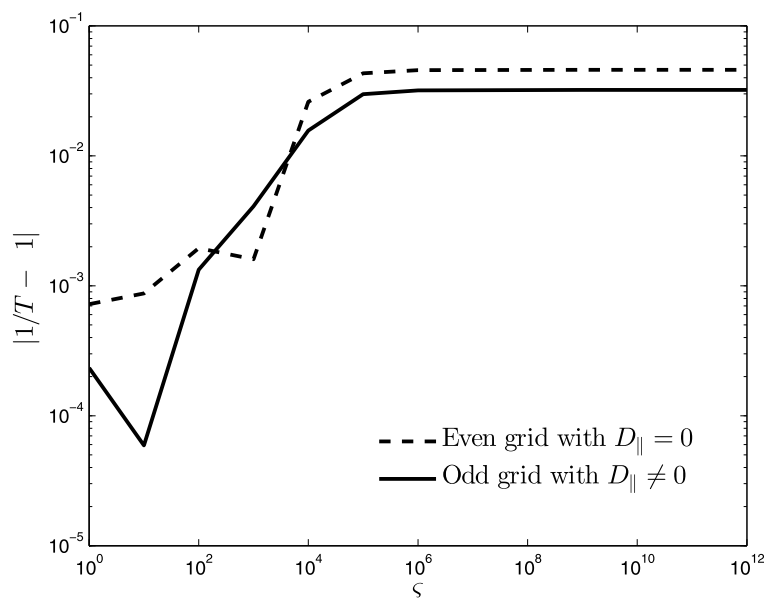

(a)

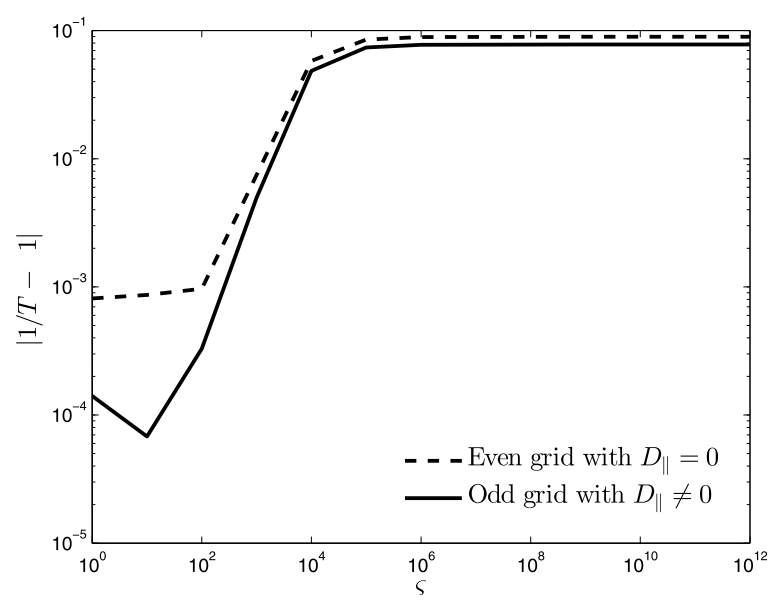

(b)

Fig. 15. Test case (10), error $\left|T^{-1}-1\right|$ with varying anisotropy for $e=\Delta s$, Vandermonde coefficients, odd and even grid with $D_{\|} \neq 0$ and $D_{\|}=0$, respectively (a) VF scheme, (b) CF scheme.

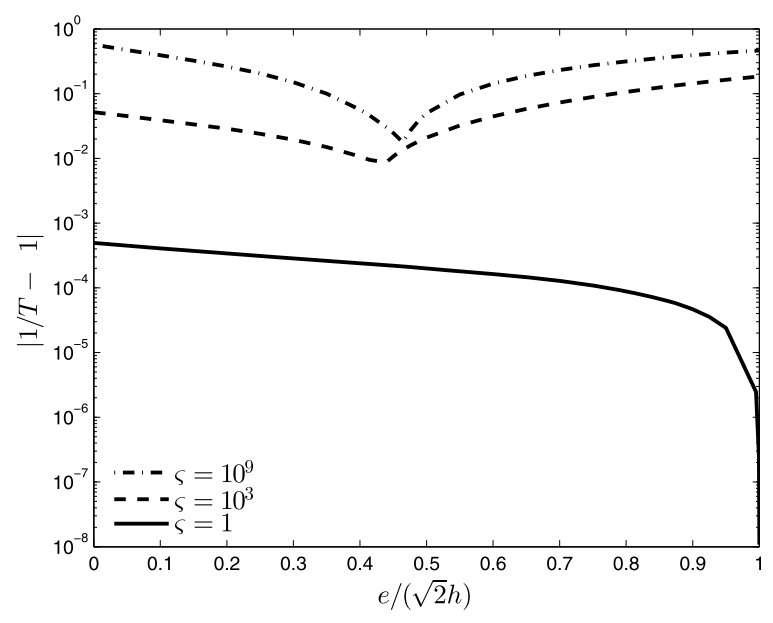

(a)

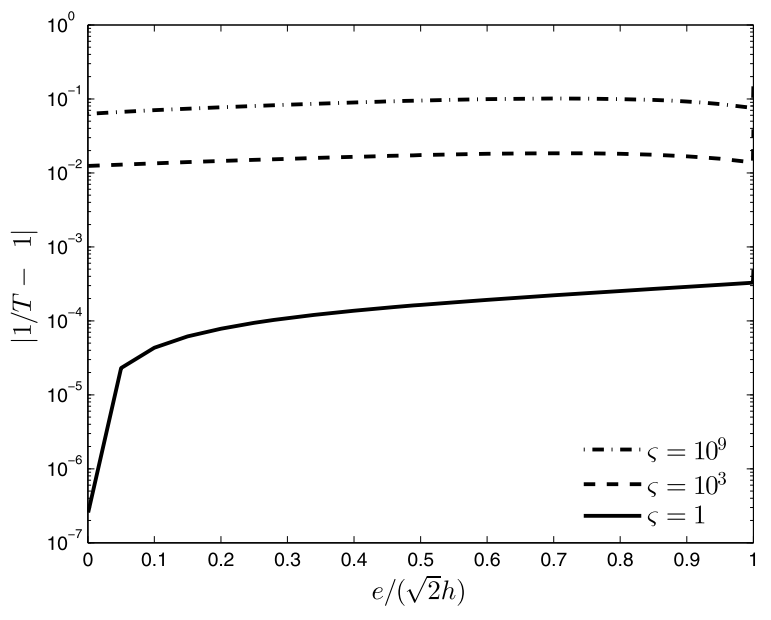

(b)

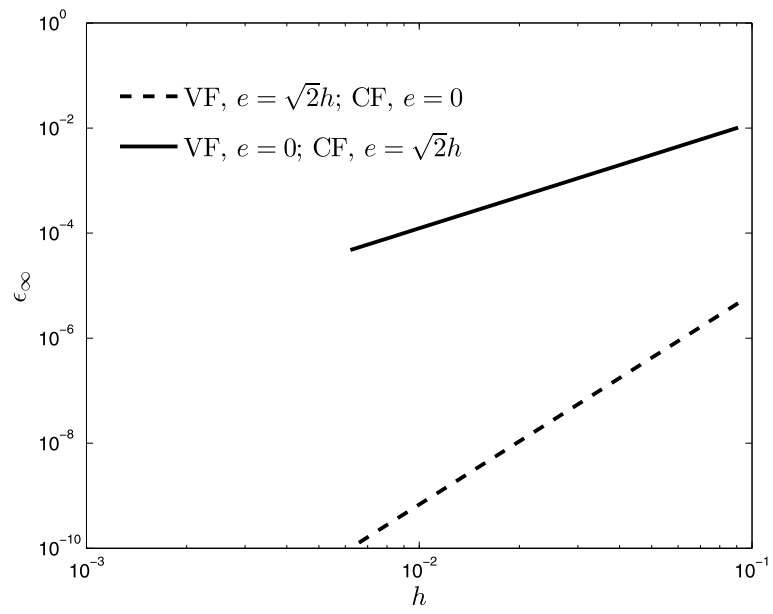

(c)

Fig. 16. Test case (10), error $\left|T^{-1}-1\right|$ for varying $e$ with anisotropy $\varsigma=1,10^{3}, 10^{9}, N=51$, Vandermonde coefficients (a) VF scheme, (b) CF scheme, (c) convergence of error $\epsilon_{\infty}$ for $\zeta=1$. 


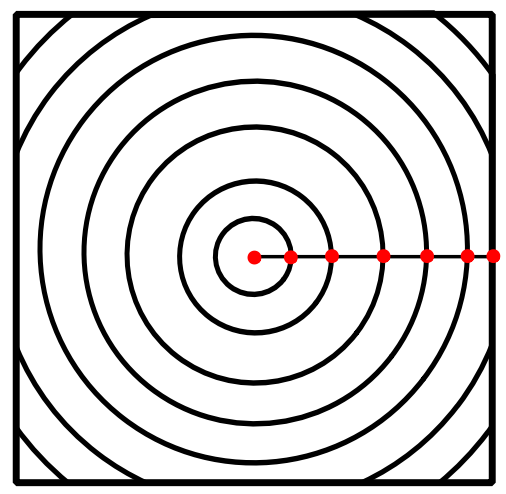

Fig. 17. Points where we set $D_{\|}$to zero.

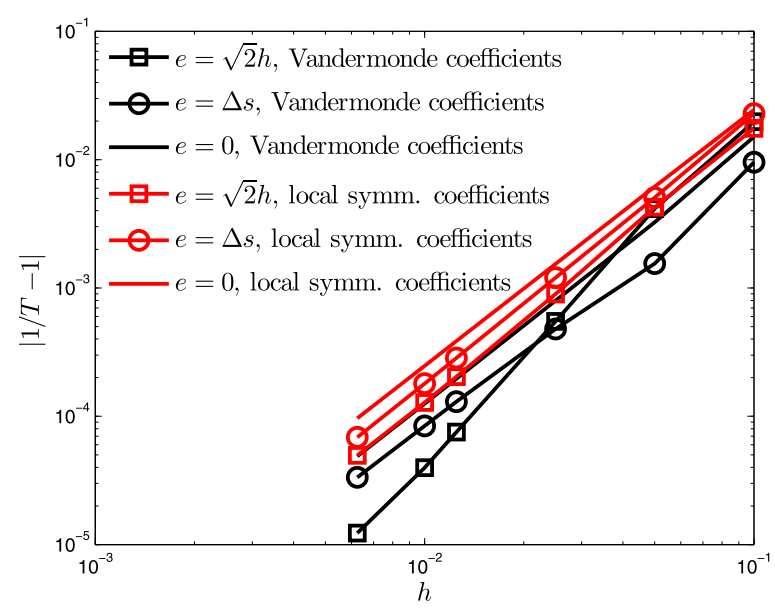

(a)

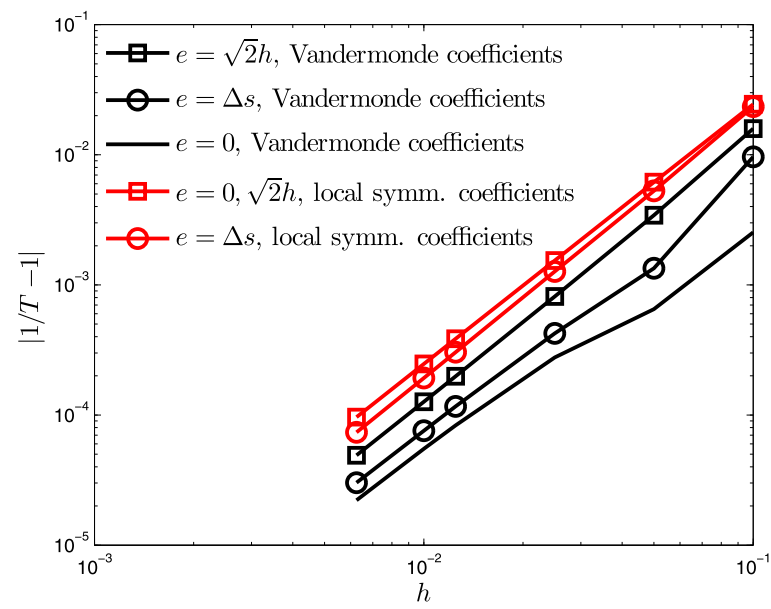

(b)

Fig. 18. Test case (10), convergence of $\left|T^{-1}-1\right|$ with $\varsigma=10^{9}$, (a) VF scheme, (b) CF scheme.

with b given by

$$
\mathbf{b}=\frac{1}{\sqrt{T_{x}^{2}+T_{y}^{2}}}\left(\begin{array}{c}
-T_{y} \\
T_{x}
\end{array}\right),
$$

which ensures that $\mathbf{b} \cdot \nabla T=0$. First we set $D_{\|}=0$ on a vertical line through the $O$-point. Second-order accuracy is obtained for $e=0$ using the VF scheme, see Fig. 19. Continuing with $e=0$ we try different enforcements of $D_{\|}=0$, from enforcement in the $O$-point only to enforcement along the full vertical. Second-order accuracy convergence for the VF scheme is obtained completely when we set $D_{\|}$to zero on the line $x=0, y>-0.5$ through the origin and likewise for $D_{\|}$set to zero on the line $x=0, y>0$, see Fig. 20. For an enforcement along the line $x=0,0<y<0.2$ stagnation of convergence sets in for a moderate resolution. In general the stagnation of convergence is delayed further for a longer line of $D_{\|}=0$ enforcement. An explanation for this is that the longer we make this line of enforcement, the more closed field lines we are able to resolve. This explains the fact that we do not see an improvement for $D_{\|}=0$ on the full vertical line compared to the half vertical line for the VF scheme since for both $x=0, y>-0.5$ and $x=0, y>0$ we treat all the closed field lines. The results for the CF scheme also improve, but there is no full recovery of the second-order accuracy. Here we should note that for $e=0$ the only difference between the CF scheme and the VF scheme is the treatment of the temperature gradients. Finally we apply the $D_{\|}=0$ enforcement on the line $x=0, y>0$ to the test case given by equation (8). Comparing the convergence results shown in Fig. 21 to those in Figs. 9, 10 we see a full anisotropy independent recovery of the convergence for $e=0, \Delta s, \sqrt{2} h$.

\subsubsection{Enforcing $D_{\|}=0$ unsteady}

To further test the influence of the closed field line adaptations discussed in section 4.3 we approximate the unsteady diffusion equation with a zero initial condition and a source function that produces the Sovinec distribution, i.e. the exact final temperature distribution and source $f$ are given by equation (10). We apply $D_{\|}=0$ on the half line of the domain 


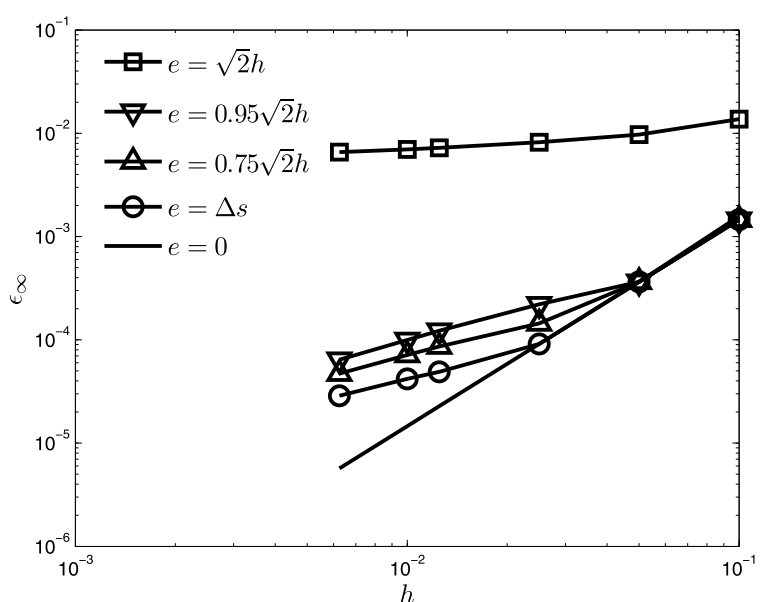

(a)

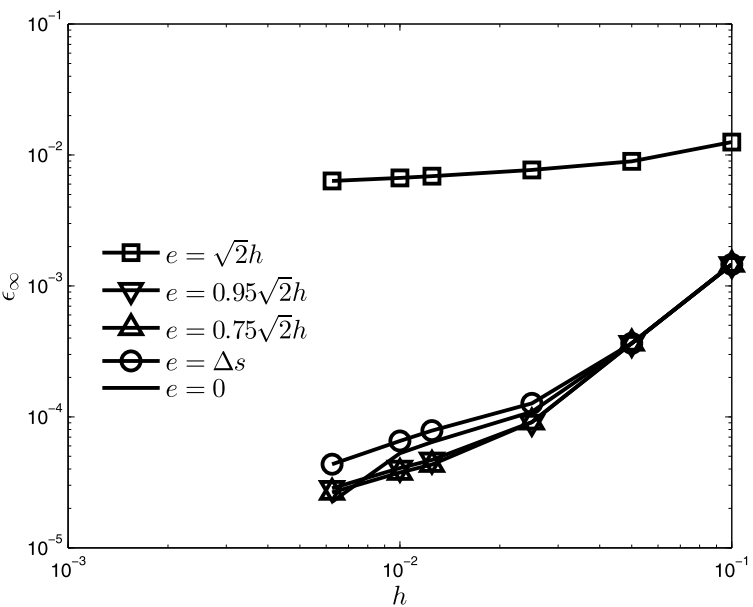

(b)

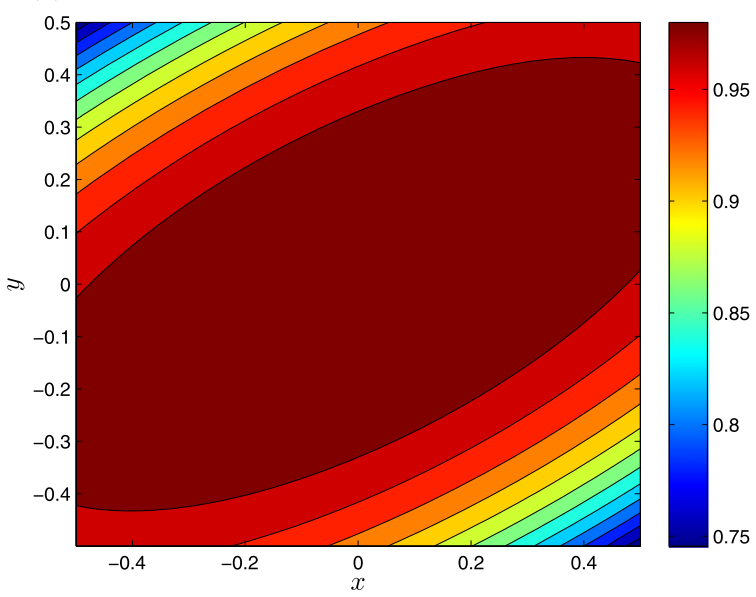

(c)

Fig. 19. Test case (11), convergence of $\epsilon_{\infty}$ with $D_{\|}$set to zero on vertical line through origin, for varying $e, \varsigma=10^{9}, \theta=1 / 3 \pi, a=0.15, b=0.85$, Vandermonde coefficients, (a) VF scheme, (b) CF scheme, (c) solution contours. (For interpretation of the colors in this figure, the reader is referred to the web version of this article.)

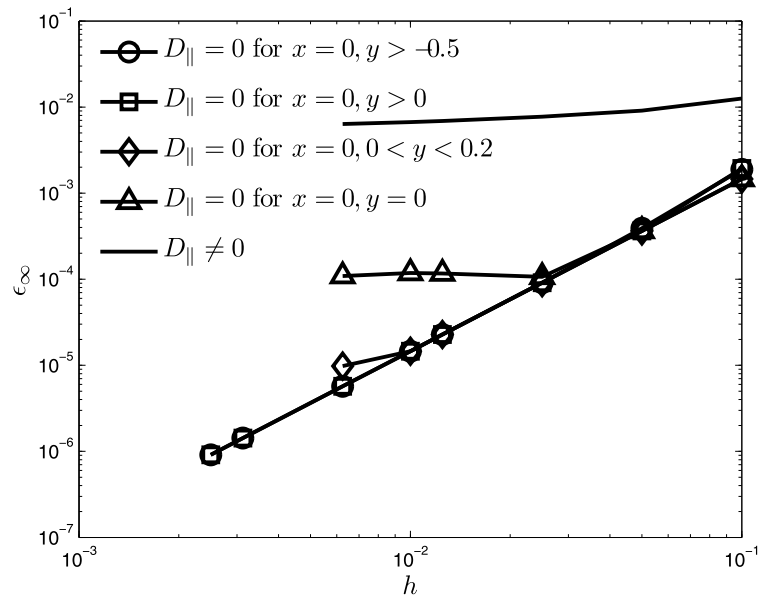

(a)

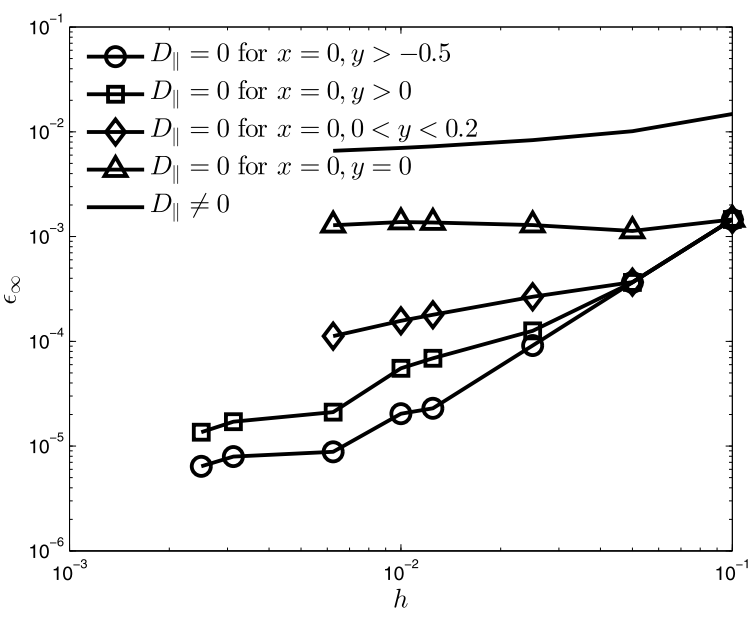

(b)

Fig. 20. Test case (11), convergence of $\epsilon_{\infty}$ for $e=0, \zeta=10^{9}, \theta=1 / 3 \pi, a=0.15, b=0.85$, Vandermonde coefficients, (a) VF scheme, (b) CF scheme. 


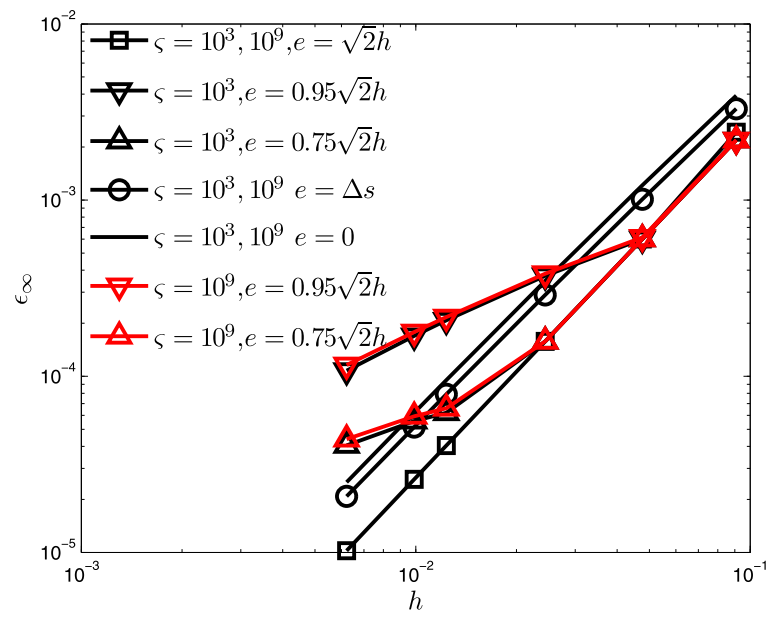

(a)

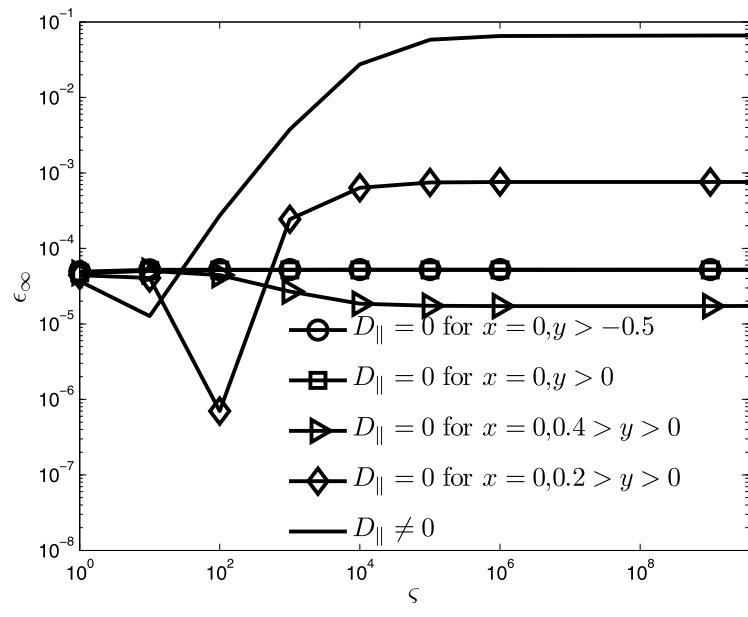

(b)

Fig. 21. Test case (8), error $\epsilon_{\infty}$ using the VF scheme with Vandermonde coefficients, (a) convergence with $\varsigma=10^{9}$ and $D_{\|}=0$ on $x=0, y>0$, (b) anisotropy dependency on $100 \times 100$ grid with $e=\Delta s$.

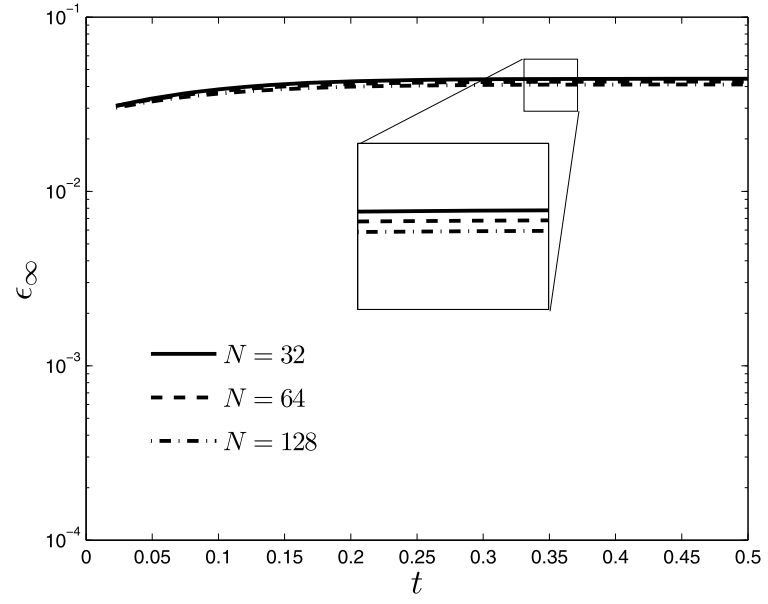

(a)

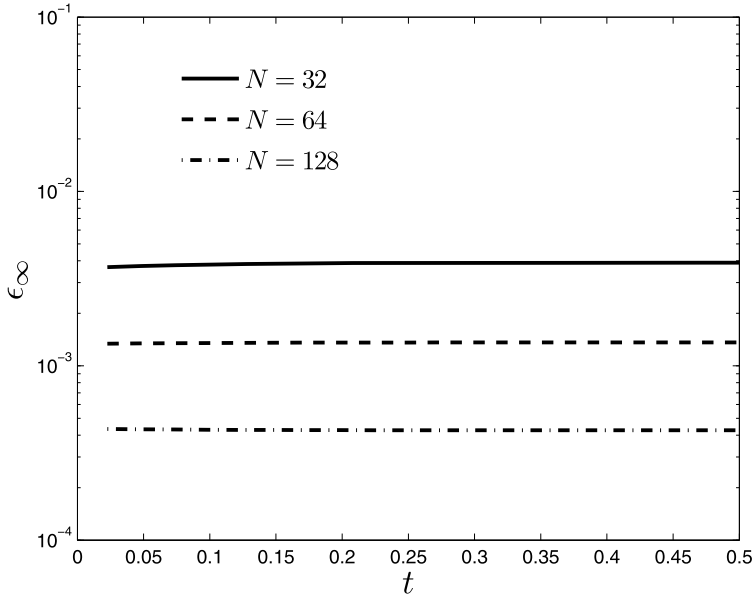

(b)

Fig. 22. Test case (12), error $\epsilon_{\infty}$ using the VF scheme with Vandermonde coefficients, $e=\Delta s, \Delta t=1 / 400, \varsigma=10^{6}$, (a) without adaptation, (b) with $D_{\|}=0$ on the half line $x=0, y>0$.

for the VF scheme with $e=\Delta s$. For the time-integration we use the Crank-Nicolson scheme. The analytical solution (see Chacón et al. [8]) is given by

$$
T(x, y, t)=\frac{1-\exp \left(-2 D_{\perp} \pi^{2} t\right)}{D_{\perp}} \cos \pi x \cos \pi y, \quad x, y \in[-0.5,0.5]
$$

In Fig. 22 we show the temporal value of the infinity error norm for an anisotropy level of $\varsigma=10^{6}$. It is clear from the figure that setting $D_{\|}$to zero on the half line greatly improves the accuracy, the accuracy for the $32 \times 32$ grid is comparable to the result by Chacón et al. [8].

\subsection{High temperature gradients}

To test the influence of high temperature gradients we apply the following temperature distribution

$$
T(x, y)=\frac{1}{1-\exp \left(-2^{1 / 2 \omega}\right)}\left[\exp \left(-2^{\omega} r^{\omega}\right)-\exp \left(-2^{1 / 2 \omega}\right)\right], \quad r^{2}=x^{2}+y^{2}, \quad x, y \in[-0.5,0.5],
$$

with $\omega$ set to a value between 2 and 6 , and again the field lines are aligned with the contours, see Fig. 23. We see from Figs. 24(a), 24(b) and 24(c) that for $\varsigma>1$ there is a significant reduction in accuracy. The current scheme does not allow for 


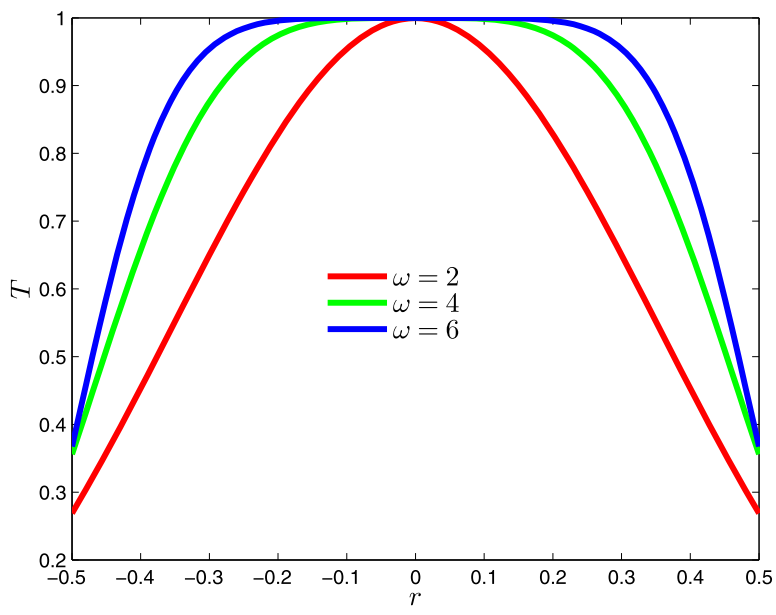

Fig. 23. Temperature distributions for high gradient test.

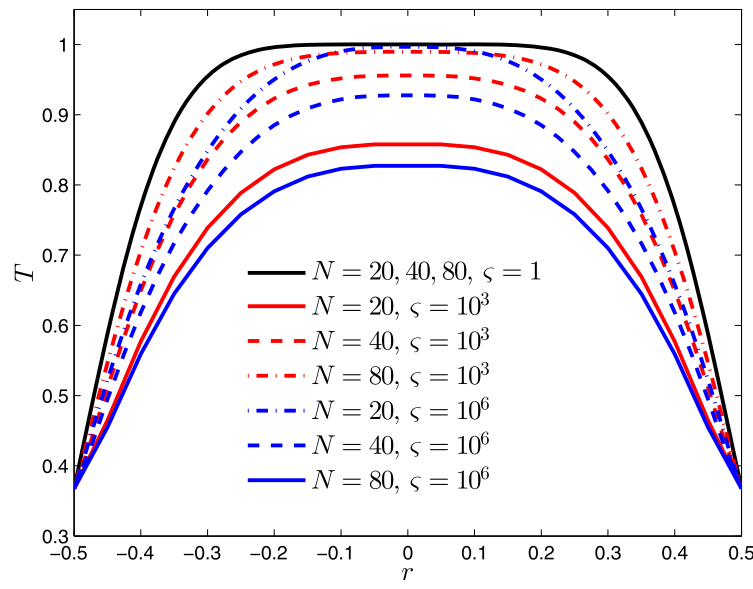

(a)

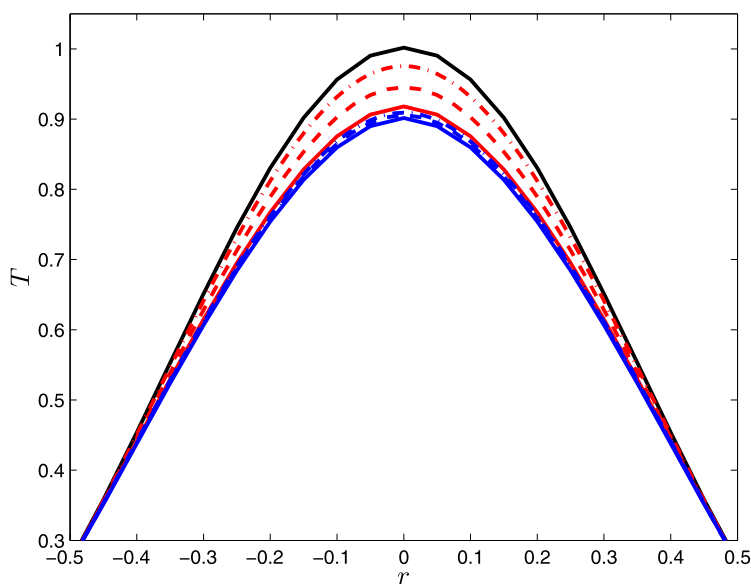

(c)

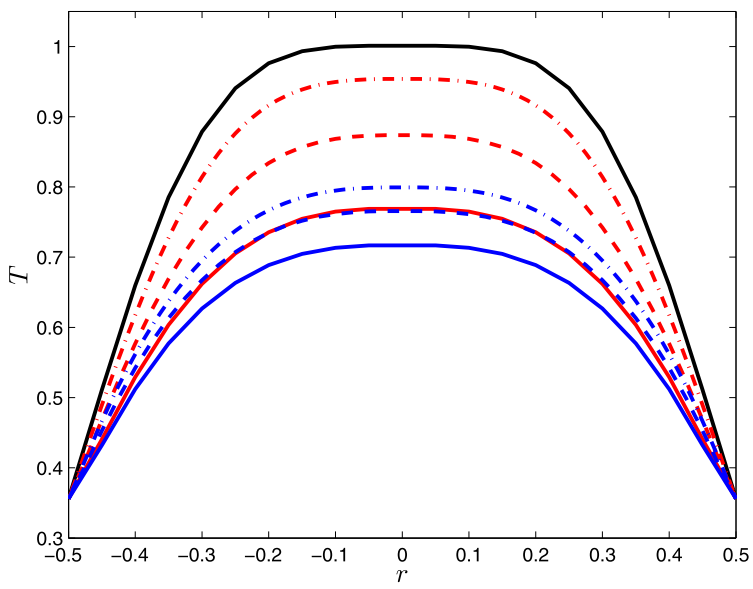

(b)

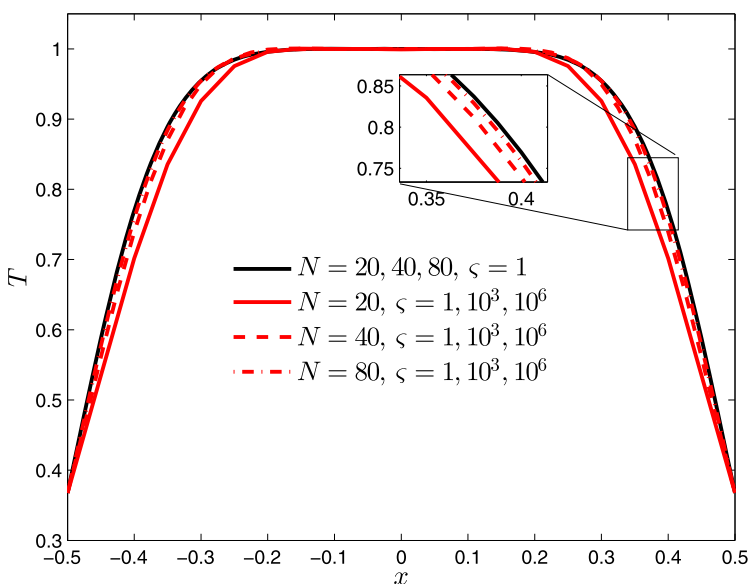

(d)

Fig. 24. Test case (13), VF scheme using Vandermonde coefficients, $e=\Delta s$ and, (a) $\omega=6$, (b) $\omega=4$, (c) $\omega=2$, (d) $\omega=6$ with $D_{\|}$set to zero on the line $x=0, y>0$.

local grid refinement for $e \in\langle 0, \sqrt{2} h\rangle$ for geometric reasons. In order to handle problems with both severe anisotropy and steep gradient regions we need to be able to align the coordinates. It is possible to use a field-aligned coordinate system. However, this cannot be maintained throughout the plasma; problems arise at $x$-points and in regions of highly fluctuating 
magnetic field directions (for instance in case of edge turbulence). Hence, the current scheme is not suitable for locally steep gradient problems as it requires an increase in overall resolution. We see in Fig. $24(\mathrm{~d})$ that applying $D_{\|}=0$ on the line $x=0, y>0$ restores the accuracy.

\section{Conclusion}

The motivation for this research was that $\mathcal{C}_{1}$ continuous connections of each volume with all of its nine neighbouring volumes would improve the accuracy for large values of the anisotropy. To have varying connectivity we introduced a parameter $e$. For $0<e<\sqrt{2} h$ we have octagonal and quadrilateral volumes connected with each other. The results indicate that connectivity plays a role in the accuracy. For all test cases the optimal result in terms of convergence was obtained for either $e=0$ or $e=\sqrt{2} h$, which is equivalent to the symmetric scheme. The connectivity as such is not of primary importance for maintaining the convergence and accuracy for the extreme anisotropy. Since a variation in $e$ does not require any regridding, it may be beneficial for an unsteady problem to adapt the value of $e$ based on previous timesteps. An extension to three dimensions is possible. The presented method is limited to smooth temperature distributions and continuous diffusion coefficient fields on Cartesian grids.

We further conclude that $D_{\|}=0$ enforcement is a viable approach to improve the tested second order finite-volume methods in case of extremely anisotropic problems with closed field lines and zero parallel diffusion. Inserting $D_{\|}=0$ on a line connecting the $O$-point with the last closed field line recovers the formal accuracy in all the test problems described in this paper, including the Sovinec test case and the tilted elliptic test case. We also conclude that having a temperature unknown exactly in the $O$-point can improve the anisotropy independence significantly in case of closed field lines. Further research is needed to verify that $D_{\|}=0$ enforcement generalizes to other approximation methods.

\section{Acknowledgements}

This work, supported by NWO and the European Communities under the contract of the Association EURATOM/FOM, was carried out within the framework of the European Fusion Program. The views and opinions expressed herein do not necessarily reflect those of the European Commission.

\section{Appendix A. Taylor expansions}

For the Asymmetric Scheme described in section 2.1 the local truncation errors of the different terms are found by writing out the Taylor expansions

$$
\begin{aligned}
& \left(D_{11} T_{x}\right)_{x}: T_{x} D_{11_{x}}+T_{x x} D_{11}+h^{2}\left[\frac{1}{24} T_{x} D_{11_{x x x}}+\frac{1}{8} T_{x x} D_{11_{x x}}+\frac{1}{6} T_{x x x} D_{11_{x}}+\frac{1}{24} T_{x x x x} D_{11}\right]+\mathcal{O}\left(h^{4}\right), \\
& \left(D_{21} T_{y}\right)_{x}: T_{y} D_{21_{x}}+T_{x y} D_{21}+h^{2}\left[\frac{1}{24} T_{y} D_{21_{x x x}}+\frac{1}{144} T_{y y y} D_{21_{x}}+\frac{1}{8} T_{x y} D_{21_{x x}}+\frac{1}{6} T_{x y y y} D_{21}\right. \\
& \left.+\frac{1}{6} T_{x x x y} D_{21}+\frac{1}{4} T_{x x y} D_{21_{x}}\right]+\mathcal{O}\left(h^{4}\right),
\end{aligned}
$$

and similarly for $\left(D_{22} T_{y}\right) y$ and $\left(D_{21} T_{x}\right)_{y}$ respectively. Likewise for the Symmetric Scheme found in section 2.2 the approximations are given by

$$
\begin{aligned}
& \left(D_{11} T_{x}\right)_{x}: T_{x} D_{11_{x}}+T_{x x} D_{11}+h^{2}\left[T_{x}\left(\frac{1}{8} D_{11_{x y y}}+\frac{1}{24} D_{11_{x x x}}\right)+T_{x x}\left(\frac{1}{8} D_{11_{y y}}+\frac{1}{8} D_{11_{x x}}\right)+\frac{1}{4} T_{x y} D_{11_{x y}}\right. \\
& \left.+\frac{1}{4} T_{x y y} D_{11_{x}}+\frac{1}{4} T_{y y x} D_{11_{y}}+\frac{1}{6} T_{x x x} D_{11_{x}}+\frac{1}{4} T_{x x y y} D_{11}+\frac{1}{12} T_{x x x x} D_{11}\right]+\mathcal{O}\left(h^{4}\right), \\
& \left(D_{21} T_{y}\right)_{x}: T_{y} D_{21_{x}}+T_{x y} D_{21}+h^{2}\left[T_{y}\left(\frac{1}{8} D_{21_{x y y}}+\frac{1}{24} D_{21_{x x x}}\right)+T_{x y}\left(\frac{1}{8} D_{21_{y y}}+\frac{1}{8} D_{21_{x x}}\right)+\frac{1}{4} T_{y y} D_{21_{x y}}\right. \\
& \left.+\frac{1}{24} T_{y y y} D_{21_{x}}+\frac{1}{16} T_{x y y} D_{21_{y}}+\frac{1}{16} T_{y x x} D_{21_{x}}+\frac{1}{6} T_{x y y y} D_{21}+\frac{1}{6} T_{y x x x} D_{21}\right]+\mathcal{O}\left(h^{4}\right),
\end{aligned}
$$

and similarly for $\left(D_{22} T_{y}\right)_{y}$ and $\left(D_{21} T_{x}\right)_{y}$ respectively.

\section{Appendix B. Interpolation coefficients}

Given an interpolation for $T$

$$
T(x, y)=\sum_{i=0}^{2} \sum_{j=0}^{2} \gamma_{i j} x^{i} y^{j},
$$


the coefficients $\gamma_{i j}$ follow from

$$
\left(\begin{array}{l}
\gamma_{22} \\
\gamma_{21} \\
\gamma_{12} \\
\gamma_{20} \\
\gamma_{02} \\
\gamma_{11} \\
\gamma_{10} \\
\gamma_{01} \\
\gamma_{00}
\end{array}\right)=\mathbf{V}^{-1} \mathbf{T}, \quad \mathbf{V}=\left(\begin{array}{ccccccccc}
h^{4} & h^{3} & -h^{3} & h^{2} & h^{2} & -h^{2} & -h & h & 1 \\
h^{4} & h^{3} & h^{3} & h^{2} & h^{2} & h^{2} & h & h & 1 \\
h^{4} & -h^{3} & -h^{3} & h^{2} & h^{2} & h^{2} & -h & -h & 1 \\
h^{4} & -h^{3} & h^{3} & h^{2} & h^{2} & -h^{2} & h & -h & 1 \\
0 & 0 & 0 & h^{2} & 0 & 0 & -h & 0 & 1 \\
0 & 0 & 0 & h^{2} & 0 & 0 & h & 0 & 1 \\
0 & 0 & 0 & 0 & h^{2} & 0 & 0 & h & 1 \\
0 & 0 & 0 & 0 & h^{2} & 0 & 0 & -h & 1 \\
0 & 0 & 0 & 0 & 0 & 0 & 0 & 0 & 1
\end{array}\right), \quad \mathbf{T}=\left(\begin{array}{c}
T_{i-1, j+1} \\
T_{i+1, j+1} \\
T_{i-1, j-1} \\
T_{i+1, j-1} \\
T_{i-1, j} \\
T_{i+1, j} \\
T_{i, j+1} \\
T_{i, j-1} \\
T_{i, j}
\end{array}\right),
$$

and are given by

$$
\begin{aligned}
& \gamma_{22}=\frac{1}{h^{4}}\left(T_{i, j}-\frac{T_{i, j-1}}{2}-\frac{T_{i-1, j}}{2}-\frac{T_{i+1, j}}{2}-\frac{T_{i, j+1}}{2}+\frac{T_{i-1, j-1}}{4}+\frac{T_{i+1, j-1}}{4}+\frac{T_{i+1, j+1}}{4}+\frac{T_{i-1, j+1}}{4}\right), \\
& \gamma_{21}=\frac{1}{4 h^{3}}\left(2 T_{i, j-1}-2 T_{i, j+1}+T_{i-1, j+1}+T_{i+1, j+1}-T_{i-1, j-1}-T_{i+1, j-1}\right), \\
& \gamma_{12}=\frac{1}{4 h^{3}}\left(2 T_{i-1, j}-2 T_{i+1, j}+T_{i+1, j-1}+T_{i+1, j+1}-T_{i-1, j-1}-T_{i-1, j+1}\right), \\
& \gamma_{20}=\frac{1}{2 h^{2}}\left(T_{i-1, j}-2 T_{i, j}+T_{i+1, j}\right), \quad \gamma_{02}=\frac{1}{2 h^{2}}\left(T_{i, j-1}-2 T_{i, j}+T_{i, j+1}\right), \\
& \gamma_{11}=\frac{1}{4 h^{2}}\left(T_{i-1, j-1}+T_{i+1, j+1}-T_{i+1, j-1}-T_{i-1, j+1}\right), \\
& \gamma_{10}=\frac{T_{i+1, j}-T_{i-1, j}}{2 h}, \quad \gamma_{01}=\frac{T_{i, j+1}-T_{i, j-1}}{2 h}, \\
& \gamma_{00}=T_{i, j},
\end{aligned}
$$

which we call the Vandermonde coefficients. Note that the coefficients $\gamma_{1}, \ldots \gamma_{8}$ are all approximations of differential terms in point $i, j$,

$$
\gamma_{i j}=\frac{1}{i ! j !}\left(\frac{\partial}{\partial x}\right)^{i}\left(\frac{\partial}{\partial y}\right)^{j} T(x, y) .
$$

\section{Appendix C. Linear operator}

For the determination of the linear operator we write,

$$
\begin{aligned}
T_{x}(x, y)= & \frac{\partial}{\partial x}\left[a_{0,0} T_{i, j}+a_{1,0} T_{i+1, j}+a_{-1,0} T_{i-1, j}+a_{0,1} T_{i, j+1}+a_{0,-1} T_{i, j-1}\right. \\
& \left.+a_{1,1} T_{i+1, j+1}+a_{-1,1} T_{i-1, j+1}+a_{1,-1} T_{i+1, j-1}+a_{-1,-1} T_{i-1, j-1}\right], \\
T_{y}(x, y)= & \frac{\partial}{\partial y}\left[a_{0,0} T_{i, j}+a_{1,0} T_{i+1, j}+a_{-1,0} T_{i-1, j}+a_{0,1} T_{i, j+1}+a_{0,-1} T_{i, j-1}\right. \\
& \left.+a_{1,1} T_{i+1, j+1}+a_{-1,1} T_{i-1, j+1}+a_{1,-1} T_{i+1, j-1}+a_{-1,-1} T_{i-1, j-1}\right],
\end{aligned}
$$

where the operators $\frac{\partial}{\partial x}, \frac{\partial}{\partial y}$ only work on the coefficients $a_{k, l}, k, l=-1,0,1$, with $x, y$ centered around $i, j$, where for the Vandermonde coefficients we have

$$
\begin{aligned}
& a_{0,0}=\frac{x^{2} y^{2}}{h^{4}}-\frac{x^{2}}{h^{2}}-\frac{y^{2}}{h^{2}}+1, \\
& a_{ \pm 1,0}=\frac{1}{2}\left(-\frac{x^{2} y^{2}}{h^{4}} \mp \frac{y^{2} x}{h^{3}}+\frac{x^{2}}{h^{2}} \pm \frac{x}{h}\right), \\
& a_{0, \pm 1}=\frac{1}{2}\left(-\frac{x^{2} y^{2}}{h^{4}} \mp \frac{x^{2} y}{h^{3}}+\frac{y^{2}}{h^{2}} \pm \frac{y}{h}\right), \\
& a_{ \pm 1,1}=\frac{1}{4}\left(\frac{x^{2} y^{2}}{h^{4}}+\frac{x^{2} y}{h^{3}} \pm \frac{y^{2} x}{h^{3}} \pm \frac{x y}{h^{2}}\right),
\end{aligned}
$$




$$
a_{ \pm 1,-1}=\frac{1}{4}\left(\frac{x^{2} y^{2}}{h^{4}}-\frac{x^{2} y}{h^{3}} \pm \frac{y^{2} x}{h^{3}} \mp \frac{x y}{h^{2}}\right) .
$$

Using the expressions for $\alpha$ given in equation (7) the contributions of the local symmetric coefficients to the linear operator are given by

$$
\begin{aligned}
\frac{\partial}{\partial x} a_{0,0} & =\frac{1}{2 h}\left(-\alpha_{r u}+\alpha_{l u}+\alpha_{l d}-\alpha_{r d}\right), & \frac{\partial}{\partial y} a_{0,0} & =\frac{1}{2 h}\left(-\alpha_{r u}-\alpha_{l u}+\alpha_{l d}+\alpha_{r d}\right), \\
\frac{\partial}{\partial x} a_{1,0} & =\frac{1}{2 h}\left(\alpha_{r u}+\alpha_{r d}\right), & \frac{\partial}{\partial y} a_{1,0} & =\frac{1}{2 h}\left(-\alpha_{r u}+\alpha_{r d}\right), \\
\frac{\partial}{\partial x} a_{-1,0} & =\frac{1}{2 h}\left(-\alpha_{l u}-\alpha_{l d}\right), & \frac{\partial}{\partial y} a_{-1,0} & =\frac{1}{2 h}\left(-\alpha_{l u}+\alpha_{l d}\right), \\
\frac{\partial}{\partial x} a_{0,1} & =\frac{1}{2 h}\left(-\alpha_{r u}+\alpha_{l u}\right), & \frac{\partial}{\partial y} a_{0,1} & =\frac{1}{2 h}\left(\alpha_{r u}+\alpha_{l u}\right), \\
\frac{\partial}{\partial x} a_{0,-1} & =\frac{x}{2 h}\left(\alpha_{l d}-\alpha_{r d}\right), & \frac{\partial}{\partial y} a_{0,-1} & =\frac{1}{2 h}\left(-\alpha_{l d}-\alpha_{r d}\right), \\
\frac{\partial}{\partial x} a_{1,1} & =\frac{\alpha_{r u}}{2 h}, & \frac{\partial}{\partial y} a_{1,1} & =\frac{\alpha_{r u}}{2 h} \\
\frac{\partial}{\partial x} a_{-1,1} & =-\frac{\alpha_{l u}}{2 h}, & \frac{\partial}{\partial y} a_{-1,1} & =\frac{\alpha_{l u}}{2 h}, \\
\frac{\partial}{\partial x} a_{1,-1} & =\frac{\alpha_{r d}}{2 h}, & \frac{\partial}{\partial y} a_{1,-1} & =-\frac{\alpha_{r d}}{2 h}, \\
\frac{\partial}{\partial x} a_{-1,-1} & =-\frac{\alpha_{l d}}{2 h}, & \frac{\partial}{\partial x} a_{-1,-1} & =-\frac{\alpha_{l d}}{2 h} .
\end{aligned}
$$

\section{References}

[1] I. Aavatsmark, An introduction to multipoint flux approximations for quadrilateral grids, Comput. Geosci. 6 (2002) 405-432.

[2] I. Aavatsmark, Multipoint flux approximation methods for quadrilateral grids, in: 9th International Forum on Reservoir Simulation, 2007.

[3] I. Aavatsmark, T. Barkve, Ø. Bøe, T. Mannseth, Discretization on non-orthogonal, curvilinear grids for multi-phase flow, in: Proc. of the 4th European Conf. on the Mathematics of Oil Recovery, Røros, vol. 6, 1994.

[4] I. Aavatsmark, T. Barkve, Ø. Bøe, T. Mannseth, Discretization on non-orthogonal, quadrilateral grids for inhomogeneous, anisotropic media, J. Comput. Phys. 127 (1996) 2-14.

[5] I. Aavatsmark, T. Barkve, Ø. Bøe, T. Mannseth, Discretization on unstructured grids for inhomogeneous, anisotropic media. Part I: Derivation of the methods, SIAM J. Sci. Comput. 19 (5) (1998) 1700-1716.

[6] I. Aavatsmark, T. Barkve, Ø. Bøe, T. Mannseth, Discretization on unstructured grids for inhomogeneous, anisotropic media. Part II: Discussion and numerical results, SIAM J. Sci. Comput. 19 (5) (1998) 1717-1736.

[7] J. Breil, P.-H. Maire, A cell-centered diffusion scheme on two-dimensional unstructured meshes, J. Comput. Phys. 224 (2007) $785-823$.

[8] L. Chacón, D. del Castillo-Negrete, C.D. Hauck, An asymptotic-preserving semi-Lagrangian algorithm for the time-dependent anisotropic heat transport equation, J. Comput. Phys. 272 (2014) 719-746.

[9] Q.-Y. Chen, J. Wan, Y. Yang, R.T. Mifflin, Enriched multi-point flux approximation for general grids, J. Comput. Phys. 3 (3) (2008) $1701-1721$.

[10] Y. Coudière, J.-P. Vila, P. Villedieu, Convergence rate of a finite volume scheme for a two dimensional convection-diffusion problem, ESAIM: Math. Model. Numer. Anal. 33 (3) (1999) 493-516.

[11] J. Droniou, Finite volume schemes for diffusion equations: introduction to and review of modern methods, HAL report hal-00813613, 2013.

[12] M.G. Edwards, M. Pal, Positive-definite q-families of continuous subcell Darcy-flux CVD(MPFA) finite-volume schemes and the mixed finite element method, Int. J. Numer. Methods Fluids 57 (4) (2008) 355-387.

[13] M.G. Edwards, C.F. Rogers, Finite volume discretization with imposed flux continuity for the general tensor pressure equation, Comput. Geosci. 2 (1998) 259-290.

[14] M.G. Edwards, H. Zheng, A quasi-positive family of continuous Darcy-flux finite-volume schemes with full pressure support, J. Comput. Phys. 227 (2008) 2152-2161.

[15] M.G. Edwards, H. Zheng, Double-families of quasi-positive Darcy-flux approximations with highly anisotropic tensors on structured and unstructured grids, J. Comput. Phys. 229 (3) (2010) 594-625.

[16] M.G. Edwards, H. Zheng, Quasi-positive families of continuous Darcy-flux finite volume schemes on structured and unstructured grids, J. Comput. Appl. Math. 234 (2010) 2152-2161.

[17] M.G. Edwards, H. Zheng, Quasi m-matrix multifamily continuous Darcy-flux approximations with full pressure support on structured and unstructured grids in three dimensions, SIAM J. Sci. Comput. 33 (2) (2011) 455-487.

[18] J.H. Ferziger, M. Perić, Computational Methods for Fluid Dynamics, Springer, 2002.

[19] H.A. Friis, M.G. Edwards, A family of MPFA finite-volume schemes with full pressure support for the general tensor pressure equation on cell-centered triangular grids, J. Comput. Phys. 230 (1) (2011) 205-231.

[20] H.A. Friis, M.G. Edwards, J. Mykkeltveit, Symmetric positive definite flux-continuous full-tensor finite-volume schemes on unstructured cell-centered triangular grids, SIAM J. Sci. Comput. 31 (2) (2008) 1192-1220.

[21] S. Günter, Q. Yu, J. Krüger, K. Lackner, Modelling of heat transport in magnetised plasmas using non-aligned coordinates, J. Comput. Phys. 209 (2005) 354-370.

[22] F. Hermeline, A finite volume method for the approximation of diffusion operators on distorted meshes, J. Comput. Phys. 160 (2000) $481-499$.

[23] M.A. Hesse, B.T. Mallison, H.A. Tchelepi, Compact multiscale finite volume method for heterogeneous anisotropic elliptic equations, Multiscale Model. Simul. 7 (2) (2008) 934-962. 
[24] J. Hyman, M. Shashkov, S. Steinberg, The numerical solution of diffusion problems in strongly heterogeneous non-isotropic materials, J. Comput. Phys. 132 (1997) $130-148$.

[25] P. Jacq, P.H. Maire, R. Abgrall, A high-order cell-centered finite volume scheme for simulating three dimensional anisotropic diffusion equations on unstructured grids, HAL report hal-00835537, 2013.

[26] P. Jenny, S.H. Lee, H.A. Tchelepi, Multi-scale finite-volume method for elliptic problems in subsurface flow simulation, J. Comput. Phys. 187 (2003) $47-67$.

[27] P. Jenny, S.H. Lee, H.A. Tchelepi, Adaptive fully implicit multi-scale finite-volume method for multi-phase flow and transport in heterogeneous porous media, J. Comput. Phys. 217 (2006) 627-641.

[28] C. Le Potier, Schéma volumes finis monotone pour des opérateurs de diffusion fortement anisotropes sur des maillages de triangles nonstructurés, C. R. Math. 341 (12) (2005) 787-792.

[29] C. Le Potier, T.H. Ong, A cell-centered scheme for heterogeneous anisotropic diffusion problems on general meshes, Int. J. Finite Vol. 8 (2012) 1-40.

[30] K. Lipnikov, M. Shashkov, D. Svyatskiy, Y. Vassilevski, Monotone finite volume schemes for diffusion equations on unstructured triangular and shaperegular polygonal meshes, J. Comput. Phys. 227 (2007) 492-512.

[31] J. Breil, P.-H. Maire, A cell-centered diffusion scheme on two-dimensional unstructured meshes, J. Comput. Phys. 224 (2) (2011) 785-823.

[32] J.E. Morel, R.M. Roberts, M. Shashkov, A local support-operators diffusion discretization scheme for quadrilateral $r-z$ meshes, J. Comput. Phys. 144 (1998) 17-51.

[33] P. Sharma, G.W. Hammett, Preserving monotonicity in anisotropic diffusion, J. Comput. Phys. 227 (2007) 123-142.

[34] M. Shashkov, S. Steinberg, Support-operator finite-difference algorithms for general elliptic problems, J. Comput. Phys. 118 (1995) $131-151$.

[35] C.R. Sovinec, A.H. Glasser, T.A. Gianakon, D.C. Barnes, R.A. Nebel, S.E. Kruger, D.D. Schnack, S.J. Plimpton, A. Tarditi, M.S. Chu, Nonlinear magnetohydrodynamics simulation using high-order finite elements, J. Comput. Phys. 195 (2004) 355-386.

[36] M.V. Umansky, M.S. Day, T.D. Rognlien, On numerical solution of strongly anisotropic diffusion equation on misaligned grids, Numer. Heat Transf., Part B 47 (2005) 533-554.

[37] B. van Es, B. Koren, H.J. de Blank, Finite-difference schemes for anisotropic diffusion, J. Comput. Phys. 272 (2014) 1-24. 